\title{
Article \\ Adaptive MPC Based Real-Time Energy Management Strategy of the Electric Sanitation Vehicles
}

\author{
Hao Wang ${ }^{1} \oplus$, Hongwen $\mathrm{He}^{1, *}{ }^{1}$, Jianwei $\mathrm{Li}^{1}$, Yunfei Bai ${ }^{1}$, Yuhua Chang ${ }^{2} \oplus$ and Beizhan Yan ${ }^{3}$ \\ 1 National Engineering Laboratory for Electric Vehicles and Collaborative Innovation Center of Electric \\ Vehicles in Beijing, School of Mechanical Engineering, Beijing Institute of Technology (BIT), Beijing 100081, \\ China; bitwanghao20@163.com (H.W.); Jianwei.li@bath.edu (J.L.); byfbit@163.com (Y.B.) \\ 2 Faculty of Automotive and Construction Machinery Engineering, Warsaw University of Technology, \\ 00-661 Warszawa, Poland; yuhua.chang@pw.edu.pl \\ 3 Yutong Bus Co., Ltd., Zhengzhou 450016, China; yanbz@yutong.com \\ * Correspondence: hwhebit@bit.edu.cn; Tel./Fax: +86-010-6891-4842
}

check for updates

Citation: Wang, H.; He, H.; Li, J.; Bai, Y.; Chang, Y.; Yan, B. Adaptive MPC Based Real-Time Energy Management Strategy of the Electric Sanitation Vehicles. Appl. Sci. 2021, 11, 498. https://doi.org/10.3390/app11020498

Received: 7 December 2020

Accepted: 3 January 2021

Published: 6 January 2021

Publisher's Note: MDPI stays neutral with regard to jurisdictional clai$\mathrm{ms}$ in published maps and institutional affiliations.

Copyright: (C) 2021 by the authors. Licensee MDPI, Basel, Switzerland. This article is an open access article distributed under the terms and conditions of the Creative Commons Attribution (CC BY) license (https:// creativecommons.org/licenses/by/ $4.0 /)$.

\begin{abstract}
Electric sanitation vehicles have increasingly been applied to cleaning work due to the requirement of air pollution control. The power distribution and energy management strategy (EMS) influence the vehicle's performance a lot both in the aspects of cleaning effect and electricity consumption. Aiming to improve energy economy and ensure clean tasks, first, the electricity consumption percentages of the vehicle onboard devices are analyzed and the main contributors are clarified, and the power requirement model of the working motor is built based on experimental data. Second, a universal modeling method of garbage distribution on the road surface is proposed, which implements a nonlinear autoregressive neural network as the predictor. Third, an adaptive model predictive control (AMPC)-based EMS is proposed and verified. The results show the AMPC method can accurately predict the garbage density and the proposed EMS can approximate the energy consumption of the DP-based EMS with little deviation. Compared to conventional rule-based EMS, the AMPC-based EMS achieved a 15.5\% decrease in energy consumption as well as a $14.6 \%$ decrease in working time.
\end{abstract}

Keywords: adaptive model predictive control (AMPC); nonlinear autoregressive neural network; electric sanitation vehicle; energy management strategy; dynamic programming

\section{Introduction}

Faced with air pollution and energy security concerns, the electrification process of conventional vehicles is overwhelming, and sanitation vehicles are no exception. The performance of an electric sanitation vehicle, which has a working motor system and a driving motor system, is strongly affected by the used energy management strategy (EMS). However, the single and rough rule-based EMS conducted by the driver fails to realize the ideal equilibrium between sanitation tasks and electricity consumption. Thus, how to elaborate an appropriate EMS, which can be implemented in real time and satisfy both clean effect and energy economy requirements, is critical in practical application.

\subsection{Literature Review and Motivation}

Recently, many EMSs have emerged to improve the energy efficiency of electric vehicles [1,2], which can be generally divided into two categories: rule-based strategies and optimization-based ones. The rule-based strategies are designed based on expert experience and can be simply implemented online [3,4], however, these deterministic methods fail to adapt to diverse driving conditions [5]. Due to a lack of sufficient optimality and adaptability, attempts to substitute or recalibrate the rule-based strategies with optimization methods were made by some researchers [3,6]. One of the most widely used optimization EMSs is dynamic programming (DP) $[7,8]$, of which the result is globally optimal and 
thus is regarded as a benchmark. Unfortunately, because of requiring full knowledge of the driving cycle and high computation burden along with great data storing demands, DP is hardly implemented in real time in vehicles. Hence, some researchers explored compromised methods such as model predictive control (MPC), which can maintain computational load within a practical range $[9,10]$. The essence of the MPC used in an EMS is to acquire sufficiently accurate predicted values related to driving cycles, which are mainly the vehicle velocity or road gradients [11] and information of transportation [11,12], to guarantee a better energy economy [13]. Consequently, tremendous research has been conducted on the prediction method [14]. Some researchers adopted the exponentially varying approximation assuming the future velocity changes exponentially [11], which aims to intuitively display how the predicted values interact with the result. Practically, this method is too generalized to adapt to the flexible velocity variation. The Markov chain is also widely used to develop stochastic MPC $[15,16]$, which predicts the future value based on the transition probability matrices. Although proven to be effective in fixed-route driving cycles, the most used 1-stage Markov chain has poor performance when confronting comprehensive driving tasks due to not including historical data. Furthermore, the artificial neural network (ANN) revealed desirable prediction accuracy $[10,13,17]$, which verified its strong capability to drive behavior pattern recognition [18], traffic flows [19], road type, and traffic congestion prediction [20]. Nevertheless, if sensitivity to the parameter tuning of ANN is not well ensured, unreliable performance might be obtained. Moreover, the autoregressive integrated moving average (ARIMA) is also taken into consideration to predict the time series [11].

All the EMSs mentioned above, especially MPC-based EMSs with different prediction methods, proved to be effective to improve the energy economy of various electric vehicles. However, the time series to be modeled and predicted featured high nonlinearity and nonstationarity, which is different from those ordinary predicted time series such as velocity profiles. Thus, the existing MPC-based EMSs may perform poorly. Moreover, to the best of our knowledge, a few existing studies, which either focused on macroscopic clean route planning [21] or the specific parameter matching [22], were related to electric sanitation vehicles.

Therefore, considering the fixed routes and environments sanitation vehicles work in, the garbage conditions on the road can be predicted. Otherwise, a dual-motor power system makes energy consumption optimization possible because the two motors have power coupled relationships from the aspects of the clean effects on the road surface. To realize an optimal power distribution on the premise that a clean task is guaranteed, the vehicle acceleration and the rotating speed of the working motor need to be optimized. Consequently, an MPC-based EMS is explored in this paper to solve the problem.

\subsection{The Organization of the Research}

The structure of the paper is organized as follows: In Section 2, the main devices onboard are modeled, and the power requirement model of the working motor is developed. A universal modeling method of practical garbage distribution on the road surface is further proposed. Section 3 proposes an AMPC-based EMS. In Section 4, the energy economy improvement and real-time feasibility of an AMPC-based EMS is substantiated through comparisons with a DP-based EMS and a rule-based EMS. Some conclusions are drawn in the final section.

\section{Modeling and Energy Consumption Analysis on an Electric Sanitation Vehicle}

The electric sanitation vehicle studied in this paper is shown in Figure 1, which was equipped with two electric motors, named the working motor and the driving motor hereafter, as shown in Figure 2. The main parameters of the vehicle are listed in Table 1. 


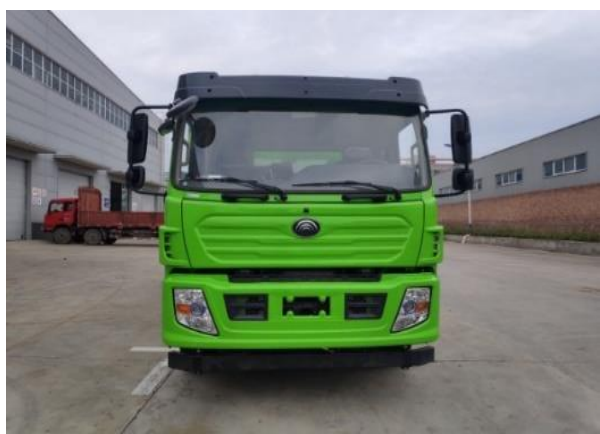

(a)

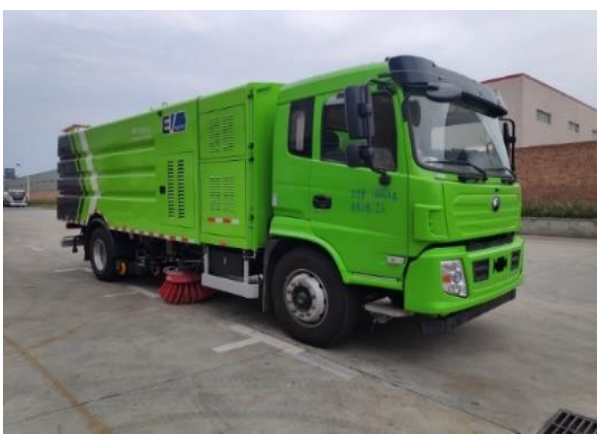

(b)

Figure 1. Research object: the sanitation vehicle: (a) front view (b) side view.

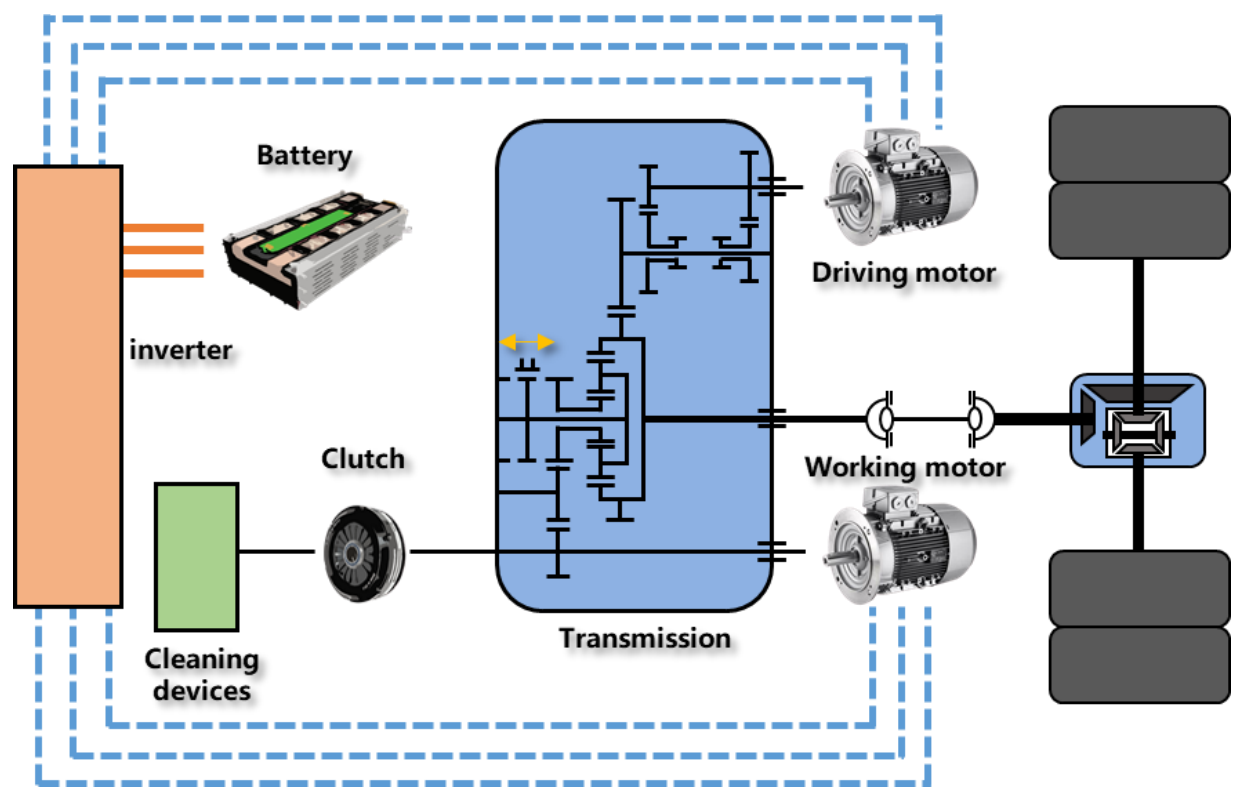

Figure 2. Structure of the electric sanitation vehicle.

Table 1. Main parameters of the sanitation vehicle insurance.

\begin{tabular}{|c|c|}
\hline Components & Description \\
\hline Electric motor 1: Driving motor & $\begin{array}{l}\text { Type: permanent magnet synchronous motor } \\
\text { Rated/Maximum power: } 100 / 184 \mathrm{~kW} \\
\text { Rated/Maximum speed: } 1600 / 5200 \mathrm{rpm} \\
\text { Rated/Peak torque: } 600 / 1100 \mathrm{Nm}\end{array}$ \\
\hline Electric motor 2: Working motor & $\begin{array}{l}\text { Type: permanent magnet synchronous motor } \\
\text { Rated/Maximum power: } 63 / 125 \mathrm{~kW} \\
\text { Rated/Maximum speed: } 2000 / 6000 \mathrm{rpm} \\
\text { Rated/Peak torque: } 300 / 650 \mathrm{Nm}\end{array}$ \\
\hline $\begin{array}{l}\text { Automated mechanical } \\
\text { transmission }\end{array}$ & $\begin{array}{l}\text { Gear ratio: }[2.5,1] \text {; Average efficiency: } 0.9 \\
\text { Final drive ratio: } 4.88 \times 2 \text { (planet set ring ratio) }\end{array}$ \\
\hline Battery & $\begin{array}{l}\text { Nominal cell voltage: } 3.2 \mathrm{~V} \text {; Total cells: } 384 \\
\text { Nominal voltage: } 614.4 \mathrm{~V} \text {; Capacity: } 254 \mathrm{Ah}\end{array}$ \\
\hline Vehicle & $\begin{array}{l}\text { Mass: } 12,000 / 18,000 \mathrm{~kg} \\
\text { Frontal area: } 8 \mathrm{~m}^{2} \text {; Air resistance coefficient: } 0.55 \\
\text { Road resistance coefficient: } 0.008 \text {; Tire radius: } 0.506 \mathrm{~m} \\
\text { Rotational mass conversion factor: } 1.1\end{array}$ \\
\hline
\end{tabular}


The electric sanitation vehicle had two operating modes: One was driving mode at high speed, the other was working mode at low speed. For the case in the working mode, the driving motor and the working motor operated separately. Namely, the torque split was not allowed through the planet sets in the transmission. The clutch was kept closed, and the working motor drove three clean devices consisting of a high-pressure water pump, a powerful vacuum collector, and a sweeper to clean the road surface. Since the particular principles of the clean process were not the point, the energy consumed by the three devices was simplified as an integration equaling that of the working motor.

\subsection{Analysis of the Energy Consumption}

Figure 3 shows the typical velocity profiles of the sanitation vehicle, which could be divided into the scenario transition condition part and the working condition part. The former part occurred in the interim when the vehicle performed such manipulations as adding water, emptying the garbage, and moving at high speed. Herein, concentrating on the working part, it is indicated that the velocity was maintained at about $8 \mathrm{~km} / \mathrm{h}$ most of the time. A slightly higher speed ranging from $10 \mathrm{~km} / \mathrm{h}$ to $22 \mathrm{~km} / \mathrm{h}$ was adopted by the vehicle when moving to the target field or turning at the road's end were required, which correspond to the segment from $2000 \mathrm{~s}$ to $2300 \mathrm{~s}$.

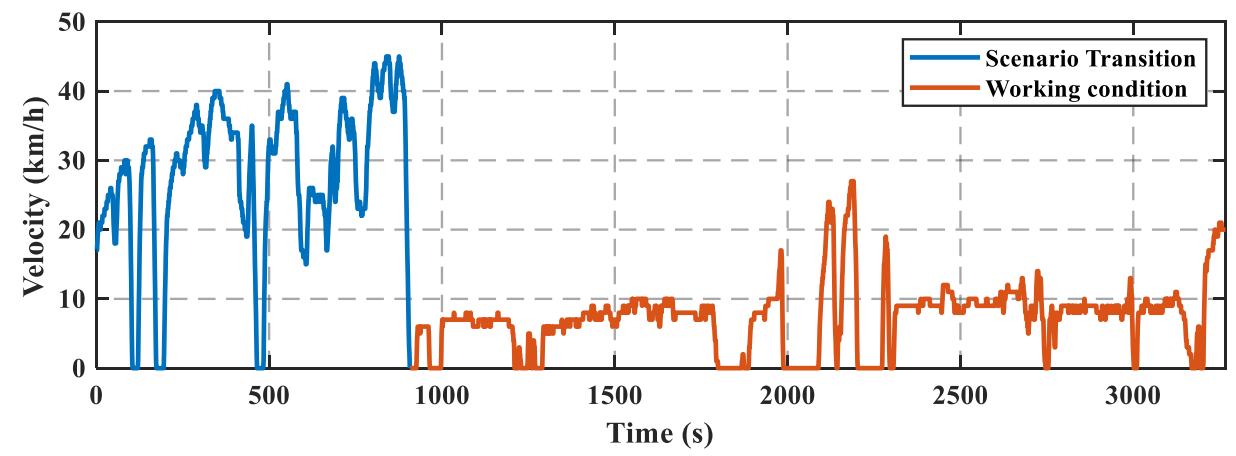

Figure 3. Typical driving cycles of the sanitation vehicle.

Although the scenario transition condition part was not of concern, the energy consumption analysis covered the whole driving cycle to reasonably evaluate the principal power components. Generally speaking, the energy stored in the battery was consumed by the driving motor (DM), the working motor (WM), an air compressor (AC) used for braking, a steering motor vehicle (SM), and a DCDC (DC-to-DC converter) connected with low-voltage accessories. Figure 4 illustrates the power curves of these components sampled from the cleaning routine. Specifically, under the driving mode, the working motor was kept off. The driving motor consumed power varying from $-120 \mathrm{~kW}$ to $120 \mathrm{~kW}$, in which being positive means that battery energy was consumed by the driving motor and being negative means that the electricity was regenerated by the driving motor from braking and restored to the battery. While under the working mode, the working motor steadily consumed power at about $50 \mathrm{~kW}$, and extremely low operating power was consumed by the driving motor due to the low velocity. In Figure 5, the operating states consisting of the rotating speed and the torque of the working motor are displayed.

In Figure 4, even though the peak power overpassed $20 \mathrm{~kW}$, it is indicated that the steering motor and air compressor merely occasionally consumed that power for a relatively short time. The energy consumption proportions of these devices are shown in Figure 6. Taking the whole driving cycle into consideration, the sum of energy consumed by the two motors took a proportion up to $89 \%$. Consequently, aiming at a better energy economy of the sanitation vehicle, only these two motors' operating states were analyzed and targeted in the optimization method to acquire optimal control sequences. 

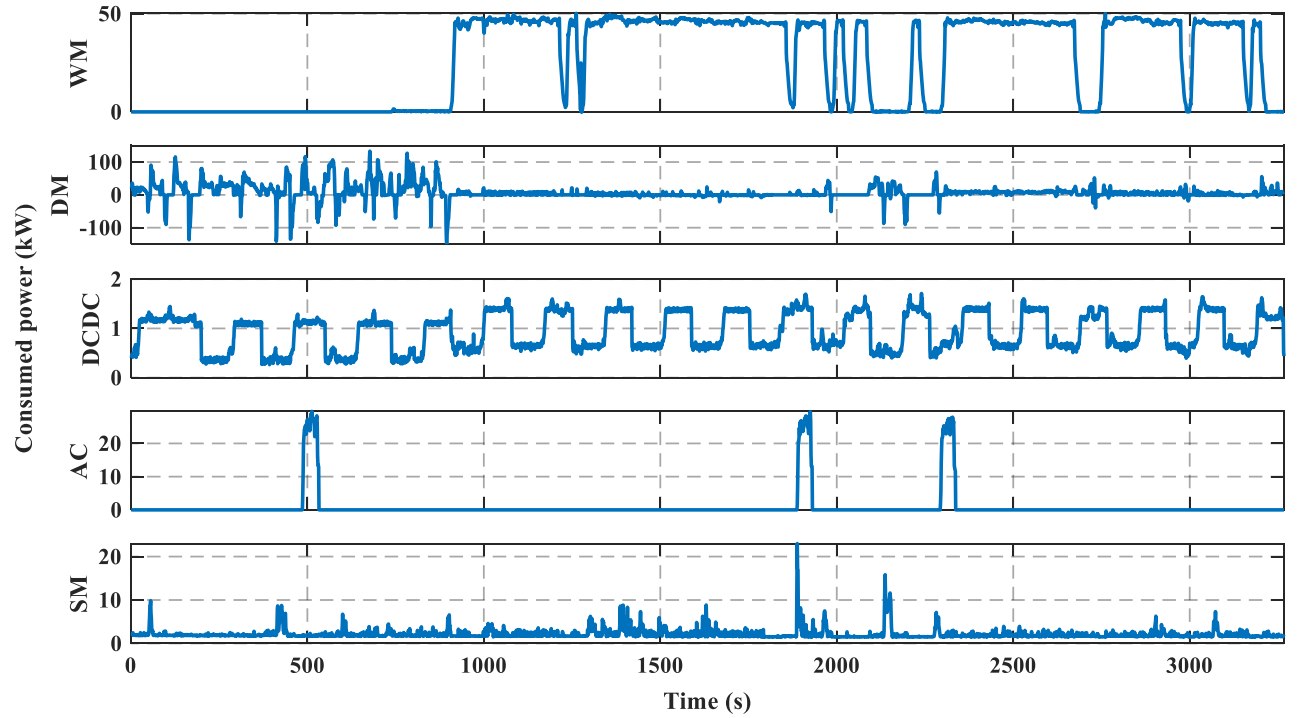

Figure 4. Electricity consumption of principal devices.

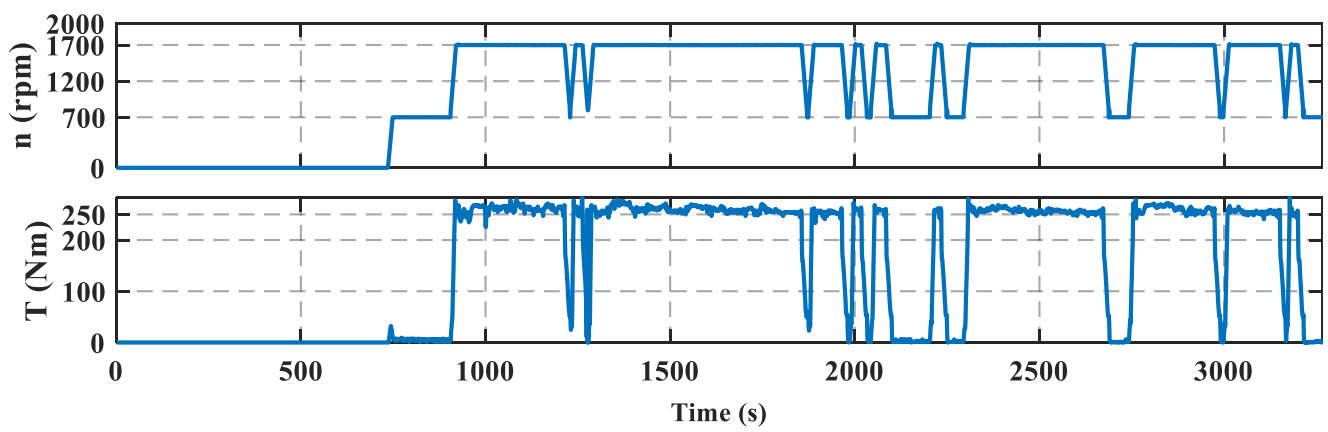

Figure 5. Operating states of the working motor.

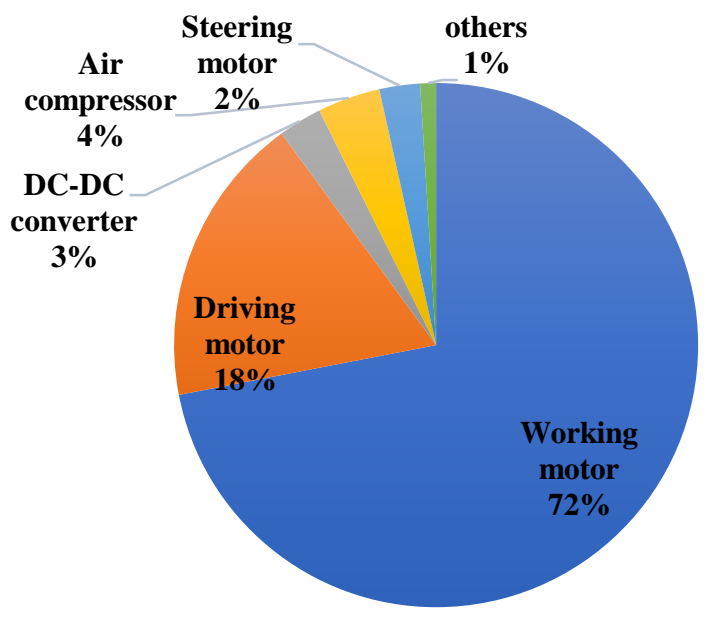
- Working motor Driving motor DC-DC converter
Air compressor $\square$ Steering motor $\square$ others

Figure 6. Energy consumption proportions of the devices onboard. 


\subsection{The Systematic Modeling}

\subsubsection{Modeling the Vehicle}

In this study, the vehicle was considered a discrete-time dynamic model. The required tractive torque $T_{d e}(\mathrm{Nm})$ and demand driving power $P_{d e}(\mathrm{~kW})$ are obtained by [23]

$$
\begin{aligned}
T_{d e} & =\frac{r}{i_{0} \cdot i_{g} \cdot \eta}\left(M g f \cos (\beta)+M g \sin (\beta)+\frac{C_{d} A}{21.15} v^{2}+\delta M a_{v}\right) \\
P_{d e} & =\frac{v}{3600}\left(M g f \cos (\beta)+M g \sin (\beta)+\frac{C_{d} A}{21.15} v^{2}+\delta M a_{v}\right)
\end{aligned}
$$

where $M$ represents the mass of the vehicle, $\mathrm{kg}$; $g$ represents the gravational acceleration, $\mathrm{m} / \mathrm{s}^{2}$; $f$ represents the rolling resistance coefficient; $A$ represents the frontal area, $\mathrm{m}^{2} ; C_{d}$ represents the air resistance coefficient; $r$ represents tire radius, $\mathrm{m} ; \delta$ represents rotational mass conversion factor; $\beta$ represents the inclination angle of the road; $a_{v}$ represents the acceleration of the vehicle, $\mathrm{m} / \mathrm{s}^{2} ; v$ represents the speed of the vehicle, $\mathrm{km} / \mathrm{h}, \eta$ represents the mechanical efficiency of the transmission system, $\delta$ represents conversion ratio of vehicle rotational mass, $i_{0}$ represents the product of final drive ratio and ring drive ratio of the planet set, and $i_{g}$ represents the transmission ratio, which was set to be the first gear due to the low velocity under working mode.

Since the vehicle was only propelled by the driving motor under the working mode, the demand torque of the vehicle approximately equaled that of the motor.

\subsubsection{Modeling the Electric Motors and Battery Pack}

Since the dynamics of the electric motor were not what this study concerned, the demand power of the two motors and the battery can be calculated by

$$
\begin{gathered}
P_{m}=\frac{T_{m} \cdot n_{m}}{9550} \\
P_{b a t}=\frac{P_{m}^{+}}{\eta_{\text {dis }} \eta_{m}}+P_{m}^{-} \eta_{c h a} \eta_{m}
\end{gathered}
$$

where $T_{m}$ represents the torque of the motor, $\mathrm{Nm} ; n_{m}$ represents rotating speed, rpm; $P_{m}$ and $P_{b a t}$ represent the demand power $(\mathrm{kW})$ of the motor and the battery, respectively; being positive $\left(P_{m}^{+}\right)$means the motors consumed electricity from the battery, and being negative $\left(P_{m}^{-}\right)$means the motors regenerated electricity to store in the battery; $\eta_{d i s}$ and $\eta_{\text {cha }}$ represents the discharging and charging efficiency of the battery, respectively; and $\eta_{m}$ stands for the motor efficiency, which could be obtained by the look-up tables of the motors shown in Figure 7.

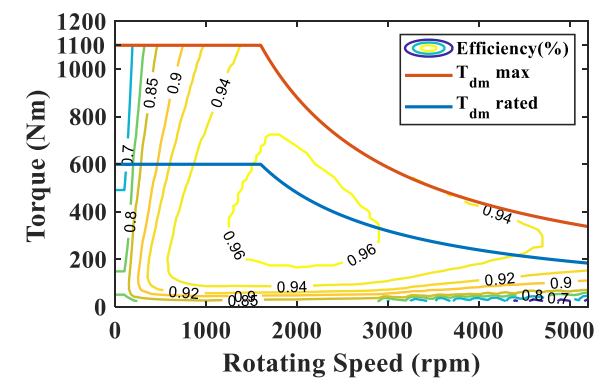

(a)

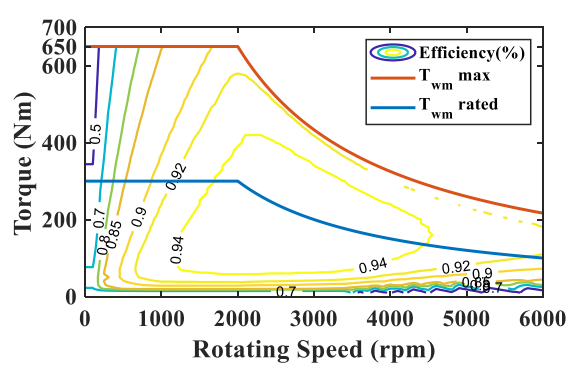

(b)

Figure 7. Efficiency map of the two motors: (a) the efficiency map of the driving motor and (b) the efficiency map of the working motor. 
Some important characteristic curves of the battery, including the open-circuit voltage and discharging resistance, are shown in Figure 8. Considering the battery health protection, the characteristics of the battery should have changed steadily [24]. Hence, the battery SoC (state of charge) was set to vary from 0.3 to 0.8 .

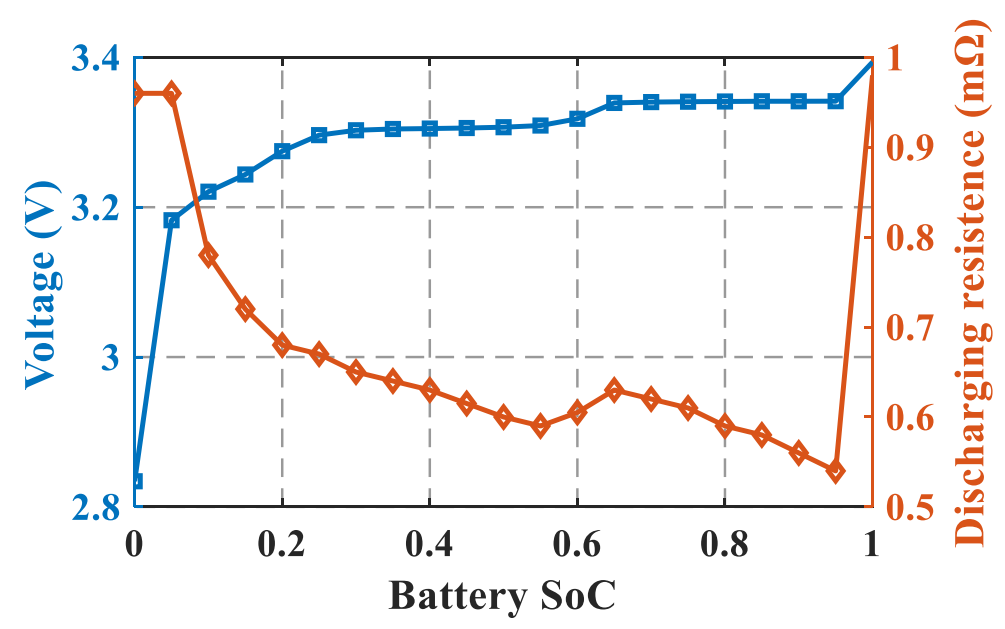

Figure 8. Characteristic curves of the battery.

Assuming that the effects of temperature on the battery are not involved, a simplified battery model is adopted with appropriate precision, which is described as [25]

$$
\frac{\mathrm{d} S o C}{\mathrm{~d} t}=-\frac{V_{b a t}-\sqrt{V_{b a t}^{2}-4 R_{b a t} P_{b a t}}}{2 R_{b a t} Q_{b a t}}
$$

where $V_{b a t}, R_{b a t}$, and $Q_{b a t}$ represent the open-circuit voltage $(\mathrm{V})$, the internal resistance $(\Omega)$, and the capacity of the battery $(\mathrm{Ah})$, respectively. Specifically, the increment of the SoC signifies the variation of vehicle electricity consumption.

\subsubsection{Modeling the Power Requirement of the Working Motor}

In practical applications, the operating state of the working motor only had three options (or three fixed rotating speeds), which are named general mode, standard mode, and extreme mode. The nominal parameters of the three modes are displayed in Table 2, which were measured when the vehicle was stationary. Since the working motor was controlled based on a certain rotating speed, the output torque changed against the resistance force from the road.

Table 2. Working motor theoretical parameters of the three modes.

\begin{tabular}{ccc}
\hline Mode Name & Fixed Rotating Speed (rpm) & Nominal Power (kW) \\
\hline General mode & 1500 & 28 \\
Standard mode & 1700 & 39 \\
Extreme mode & 1900 & 54 \\
\hline
\end{tabular}

To investigate how garbage density on the road interacts with vehicle energy consumption, a series of field experiments, which were devised based on actual operations of drivers, were conducted to establish the power requirement model of the working motor. The real garbage was replaced with sand and rubble in the experiments.

Figure 9 displays the main experimental results. It is indicated that the residues after cleaning were basically consistent or even less, despite having more and more "garbage" on the road surface. From Figure 9, it seems the "garbage" could not be cleaned completely. However, due to far less existing garbage in real scenarios, hardly any residues would be 
left after cleaning. Setting the quantity of "garbage" in experiments to be significantly more than the real would achieve a clearer visual comparison.

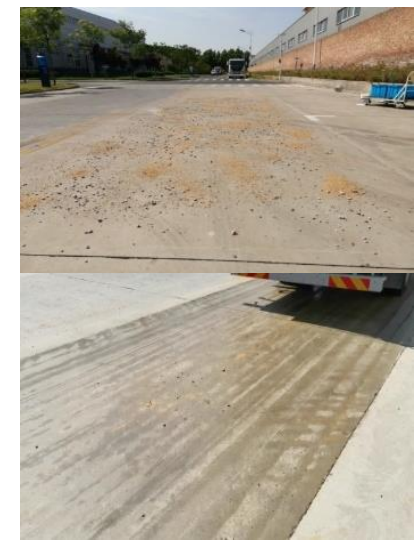

(a)

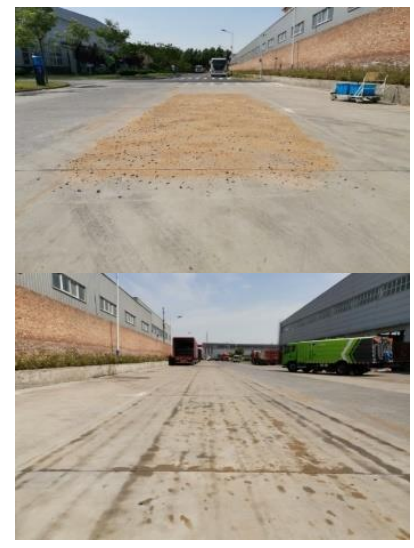

(b)

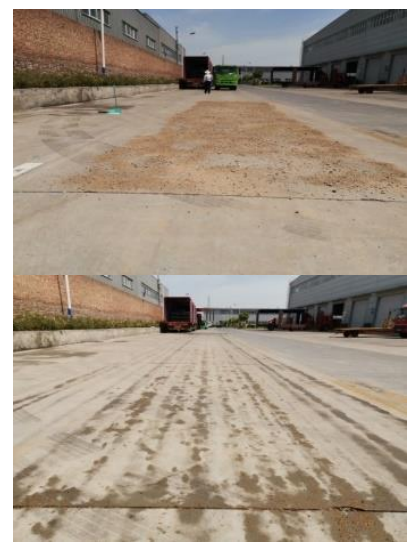

(c)

Figure 9. Field experiment results: (a) under general mode, (b) under standard mode, and (c) under extreme mode.

The detailed results of the experiments are summarized in Table 3, where the performance was evaluated by the proportions of residues in the total amount of garbage.

Table 3. Experiment results.

\begin{tabular}{|c|c|c|c|c|}
\hline Working Mode & Vehicle Velocity $(\mathrm{km} / \mathrm{h})$ & Motor Rotating Speed (rpm) & Garbage Density $\left(\mathrm{g} / \mathrm{m}^{2}\right)$ & Performance $(\%)$ \\
\hline General mode & 8 & 1500 & $\begin{array}{l}200 \mathrm{~g} \text { sands }+ \\
200 \mathrm{~g} \text { rubbles }\end{array}$ & 97.3 \\
\hline Standard mode & 8 & 1700 & $\begin{array}{l}400 \mathrm{~g} \text { sands }+ \\
200 \mathrm{~g} \text { rubbles }\end{array}$ & 98.5 \\
\hline Extreme mode & 8 & 1900 & $\begin{array}{l}600 \mathrm{~g} \text { sands }+ \\
200 \mathrm{~g} \text { rubbles }\end{array}$ & 99 \\
\hline Maximum ability test & 4 & 1900 & $\begin{array}{l}1500 \mathrm{~g} \text { sands }+ \\
200 \mathrm{~g} \text { rubbles }\end{array}$ & 97.1 \\
\hline
\end{tabular}

As mentioned before, the working motor was controlled based on rotating speed, which is formulated as

$$
T_{\text {wm }}=f\left(n_{w m}, F_{\text {resist }}\right)
$$

where $T_{w m}$ is the working motor output torque, $n_{w m}$ is the rotating speed, and $F_{\text {resist }}$ represents the resistance from the road surface, which is theoretically related to the velocity, garbage density, and the rotating speed of the working motor.

Based on the experiment data, it can be summarized that the variations of vehicle speed and garbage density exerted very limited impact on the motor torque, which is also testified in Figure 5. Consequently, assuming being immune from those minor factors, the relationship between power and the rotating speed of the working motor could be approximated mathematically.

Furthermore, it is evident the clean force, which ranged from $0 \sim 1$, representing normalized cleaning ability of the vehicle, increased with the power of the motor but decreased with the vehicle velocity. Hence, the discount factor $\gamma$ dependent on velocity is defined and the cleaning effect of the vehicle is formulated as

$$
\psi_{\text {res }}=\delta_{\text {garbage }} \cdot\left(1-\gamma(v) \cdot \phi\left(P_{w m}\right)\right)
$$


where $\delta_{\text {garbage }}$ represents the garbage density on the road, $\mathrm{g} / \mathrm{m}^{2} ; \gamma$ represents the discount factor; $\phi($.$) represents the functional relationship between clean force and motor power;$ and $\psi_{\text {res }}$ represents the garbage residue, $\mathrm{g} / \mathrm{m}^{2}$.

All functional relationships, which include $f($.$) in Equation (6) and \phi(),. \gamma($, ) in Equation (7), are modeled with the non-linear least square algorithm. The modeling method is formulated as:

$$
\begin{gathered}
\min \{S\}: S=\sum_{i=1}^{m}\left(y_{i}-f\left(x_{i}, \beta\right)\right)^{2} \\
\beta=\left(\beta_{1}, \beta_{2}, \ldots, \beta_{n}\right)
\end{gathered}
$$

where $m$ is the number of samples, $y_{i}$ is the real value, $f($.$) represents the relationships to$ be established, and $\beta$ is a vector whose elements are decided by minimizing the $S$.

The power requirement model of the working motor is illustrated in Figure 10.
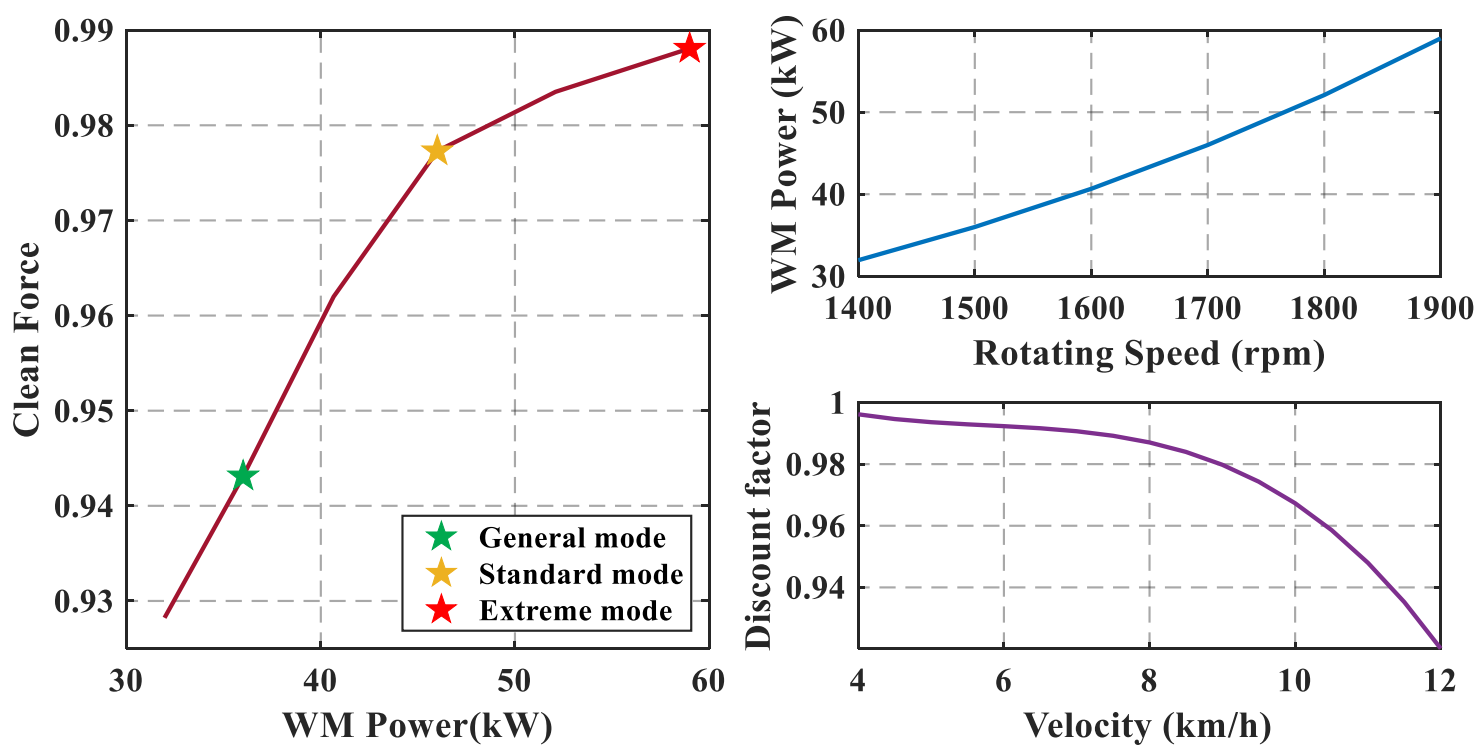

Figure 10. Power requirement model of the working motor.

\subsubsection{Modeling the Garbage Distribution on the Road Surface}

Conventionally, garbage density with the unit as $\mathrm{kg} / \mathrm{m}^{2}$ is used to describe the amount of garbage, which is a broad category including waste and dust. The target street where the sanitation vehicles work was a typical one stretching from downtown to the suburb. Through field observation, it was concluded that the garbage density distribution was significantly influenced by the green belts, which periodically appeared along the roadside, bringing fallen plant tissues, and commercial regions with strong fluidity. Thus, the garbage distribution model was established as the following steps:

Step 1: cleaning levels ranging from 1 to 4 were defined to preliminarily classify the different segments of the road. Segments labeled with the high cleaning level had more garbage. Specifically, level 1 aimed at relatively clean segments without green belts alongside; level 2 was applied to the segments with some fallen tissues or garbage; then, segments marked level 3 had more garbage or leaves that were simple to clean. However, there were more or less some segments covered by materials such as oil stains or squashed dirt, making it tougher to wash thoroughly, for which the highest level 4 was defined. Some examples are shown in Figure 11. 


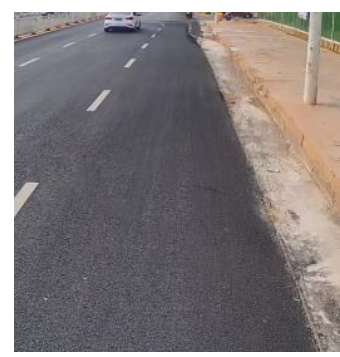

(a)

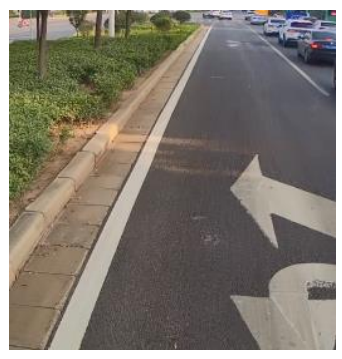

(b)

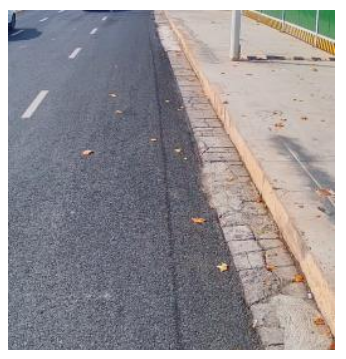

(c)

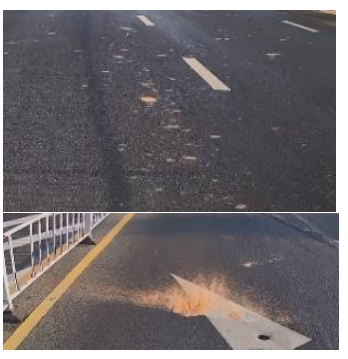

(d)

Figure 11. Examples for segments marked with different levels: (a) Level 1, (b) Level 2, (c) Level 3, and (d) Level 4.

Step 2: The garbage density distribution was a result of multiple factors involving both natural and artificial influence. However, a qualified model could be established based on the fact that garbage density was probably continuous and followed historical patterns. Therefore, statistically, the garbage density on a certain level of segment tended to be normally distributed, whose mean value and standard deviation could be assumed reasonably.

Step 3: A certain garbage density value generated from the normal distribution model in step 2 was probably maintained for tens of meters on the segment, which could similarly be estimated based on the actual condition.

Step 4 resulted from multiple uncertain factors, and the fluctuation of the garbage density was inevitable. Hence, different sets of white Gaussian noise were added to the garbage model to reflect the randomness of the real scenario.

The modeling process is illustrated by a flow chart shown in Figure 12.

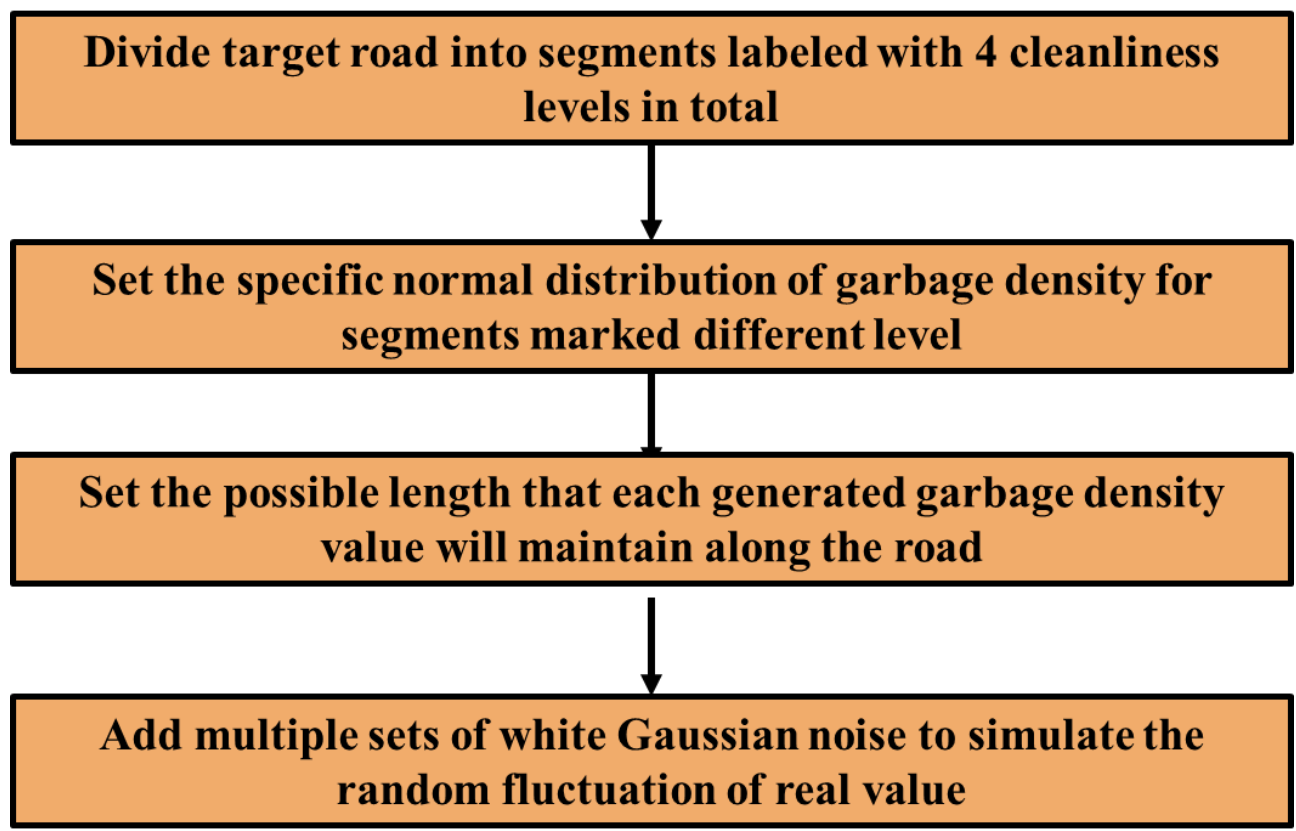

Figure 12. Modeling the process of the garbage distribution.

Figure 13 displays one of the possible garbage distribution models. It is worth noting that, apart from segment levels 1 to 3 that were affected by regular factors such as artificial green belts, the segments marked level 4 were almost unforeseeable, occurring less but more randomly and continuing for a much shorter distance in practice. 


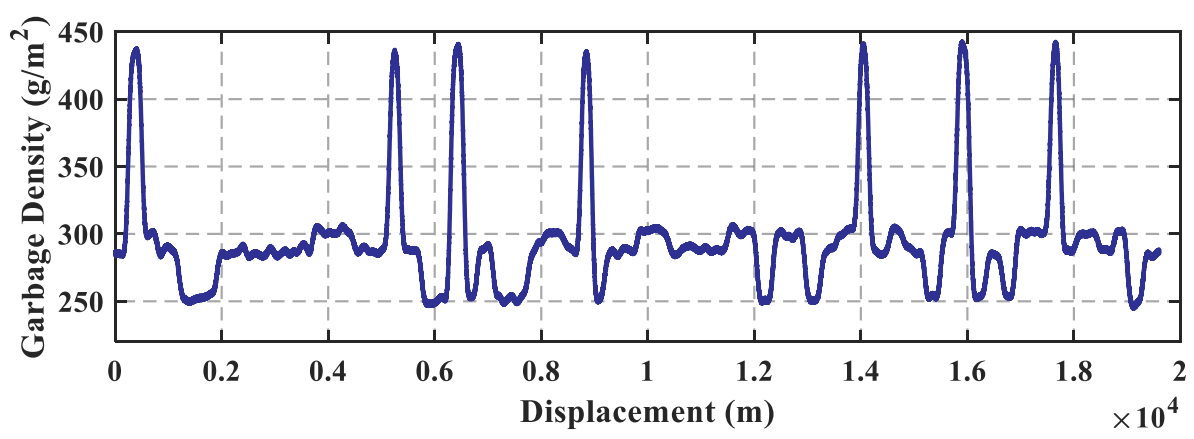

Figure 13. Garbage density distribution model.

\section{The Real-Time AMPC-Based EMS}

\subsection{The Prediction Method}

Since the vehicle was clean task-oriented, any optimal manipulations were radically decided by the garbage density ahead on the road surface. Considering the vehicle velocity was almost constantly under the working mode, the garbage density sequence in the spatial domain could be equivalently regarded as a time series. Therefore, on the spatial prediction horizon, the predictor could anticipate the garbage density series with the methods dealing with time series. To make the description uniform, the rest of this study discusses problems from the perspective of the spatial domain.

Due to the high autocorrelation of the garbage density distribution, the future values could be forecasted using the past values in the same series by the predictor. The autoregressive relationship to be modeled can be formulated as

$$
\delta(k)=f(\delta(k-1), \delta(k-2), \ldots, \delta(k-p))+\varepsilon_{t}
$$

where $\delta(k)$ represents the value of the garbage density series at displacement $k ; \delta(k-1)$, $\delta(k-2), \ldots$ represent the past values of the series; $f($.$) represents the unknown non-linear$ relationships to be approximated, $p$ represents the number of past values, and $\varepsilon_{-} t$ stands for the error of the approximation.

However, for the garbage density series, the relationships $f($.$) might have varied with$ different segments of the road, which made the predictors using a Markov chain [15] or ARIMA [11] suitable for nonstationary "time" series perform poorly. Hence, the non-linear autoregressive neural network (NARNN) was implemented, which deals effectively with "time" series characterized by frequent variations and high nonstationarity. The discrete, non-linear, autoregressive model build by NARNN can be precisely written as [26]:

$$
\delta(k)=\alpha_{0}+\sum_{j=1}^{m} \alpha_{j} \phi\left(\sum_{i=1}^{p} \beta_{i j} \delta(k-i)+\beta_{0 j}\right)+\varepsilon_{t}
$$

where $p$ represents the number of the past values (or delays) used as the input to predict; $m$ represents the number of hidden layer neurons in NARNN with the activation function defined as $\phi($.$) -specifically, each neuron can approximate a unique relationship f(.) ; \beta_{i j}$ represents the parameter corresponding to the weight of the connection between the input neural cell $i$ and the hidden cell $j ; \alpha_{j}$ stands for the weight of the connection between the hidden cell $j$ and the output cell; and $\beta_{0 j}$ and $\alpha_{0}$ are the constants respectively corresponding to the hidden cell and the output cell. The general working principle of the predictor is shown in Figure 14. 


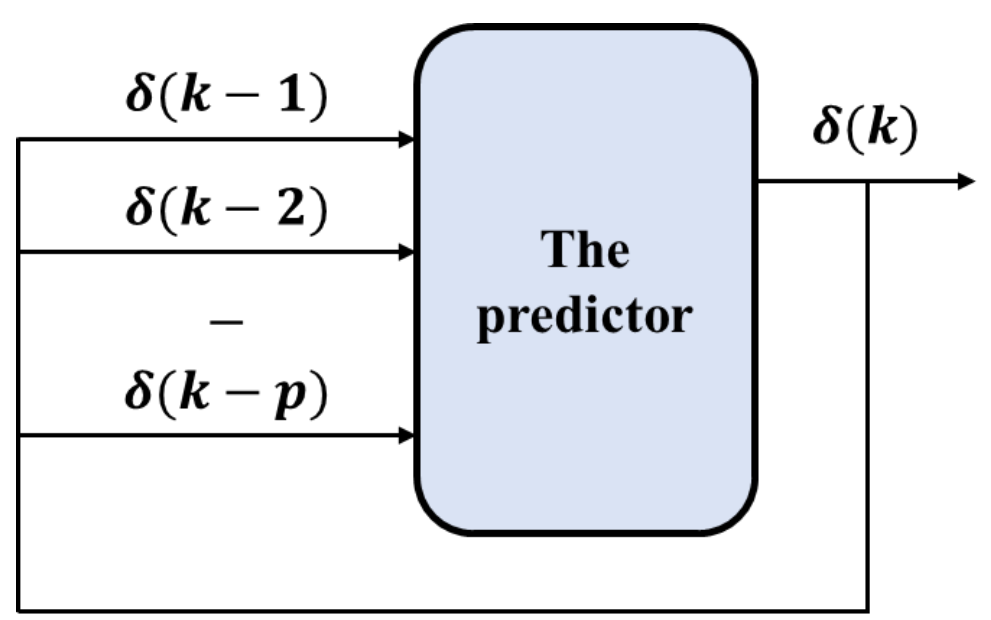

Figure 14. The topology of the NARNN predictor.

To guarantee the calculation efficiency, the learning rule of the NARNN uses the Levenberg-Marquardt backpropagation-type algorithm procedure (LMBP), which is regarded as the fastest of the category. Furthermore, the number of hidden layers and neurons per layer for NARNN is completely flexible and can be optimized through a trial-and-error procedure to get the best performance [27].

Increasing the number of neurons helps the NARNN simulate more complex relationships and improve the generalization ability, while decreasing that number alleviates the computation burden and contributes to the real-time requirement. Consequently, the NARNN was set to have 20 neurons in 1 hidden layer, which could well trace the variation tendency of the garbage density, and the delay of the spatial series was set to be 11 steps optimally. Established on the Matlab platform, the open-loop training process of the NARNN is shown in Figure 15.

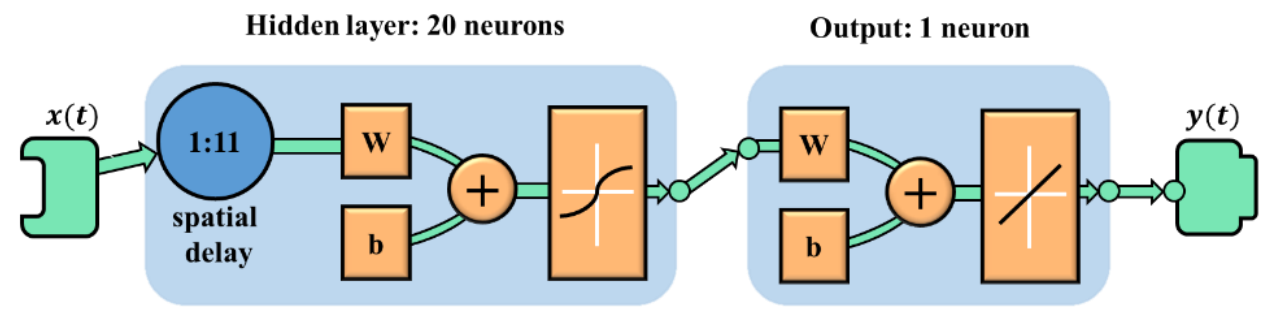

Figure 15. The training process of the NARNN.

It is critical to bear in mind that the essence of ensuring the performance of a neural network is whether broad possible instances are covered in the training set. Since the garbage distribution model constituted segments involving all likely scenarios, the training set was generated from the former $75 \%$ of data in the established model (Figure 13). Thus, the parameters of the NARNN predictor mentioned in the Equation (11) could be updated with supervised learning. The performance of the trained neural network is shown in Figure 16 and the final precision was appropriate, with a mean square error (MSE) equaling 1.35. Details of training performance of NARNN predictor are shown in Figure 17. 


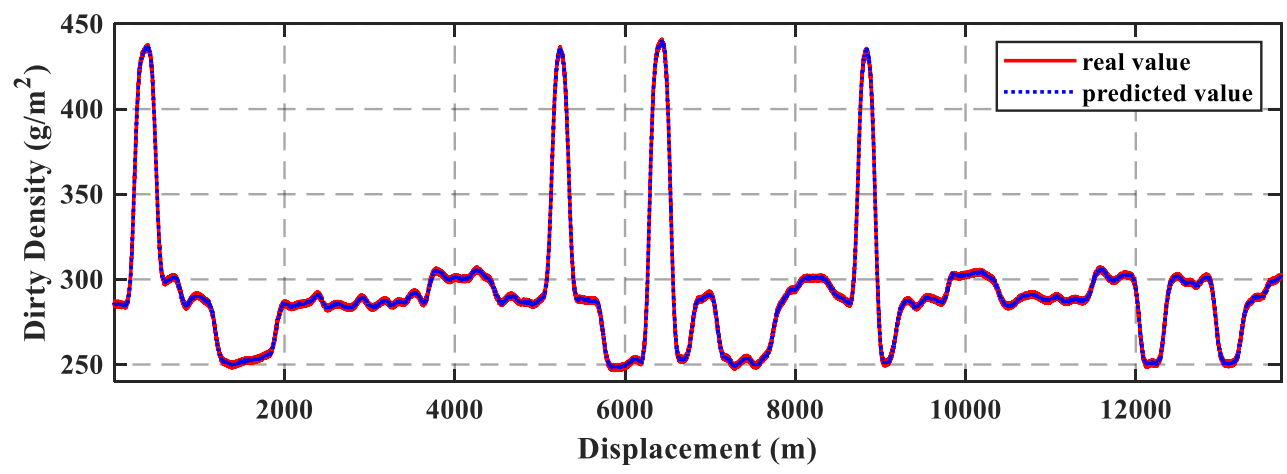

Figure 16. Training verification of the NARNN.

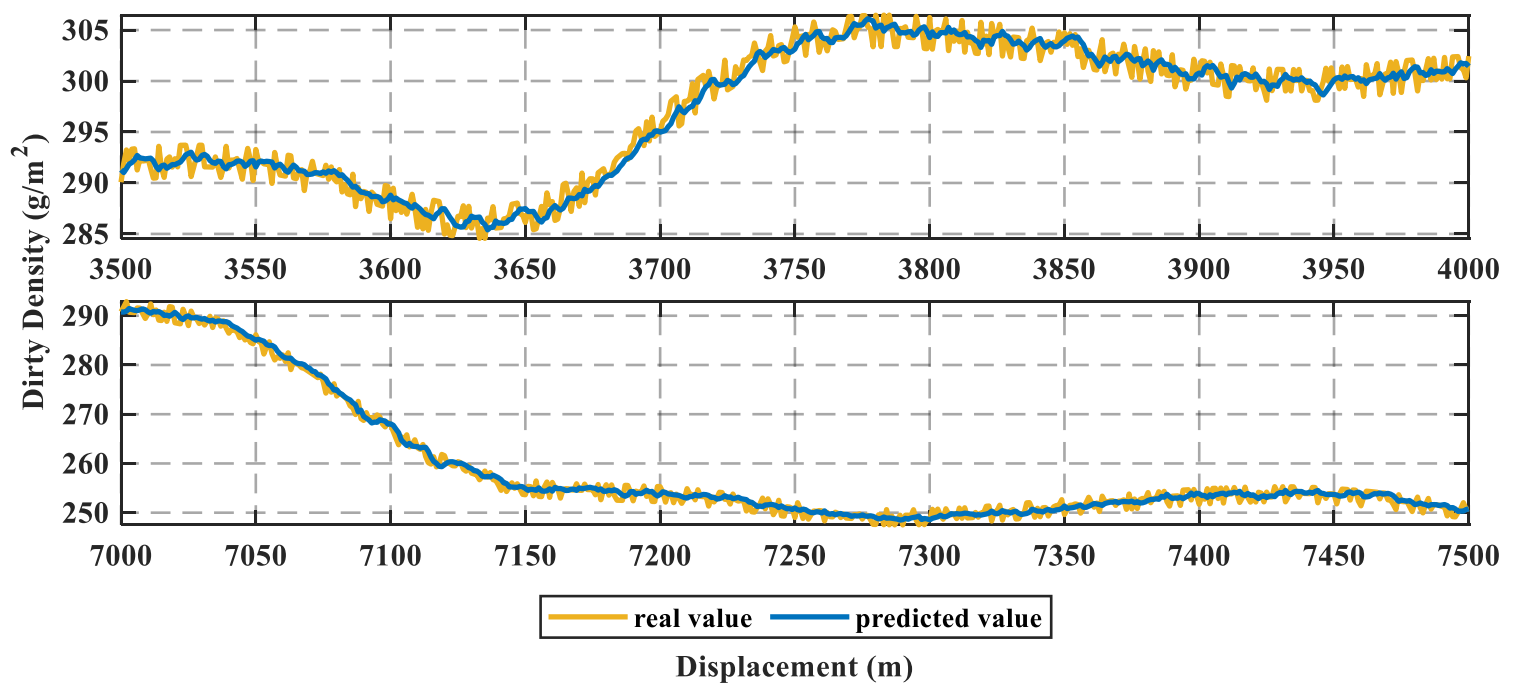

Figure 17. Details of training performance of the NARNN.

After the open-loop training was accomplished, as Figure 18 displays, the network was converted to a closed loop for prediction. Supplied with the initial $p$ values, the future garbage density $\delta(k+1), \delta(k+2), \ldots \delta(k+L)$ series could be iteratively forecasted using the latest past $p$ values, including the predicted ones [28]. Nevertheless, with the increase of prediction horizon length, the error inevitably accumulates because of the iteration process. To restrain it within an acceptable range, the NARNN predictor updates the past values with the real detected ones as the prediction horizon moves forward, which can be realized with a visual sensor used to estimate the garbage density. The length of the prediction zone was set to be $20 \mathrm{~m}$.

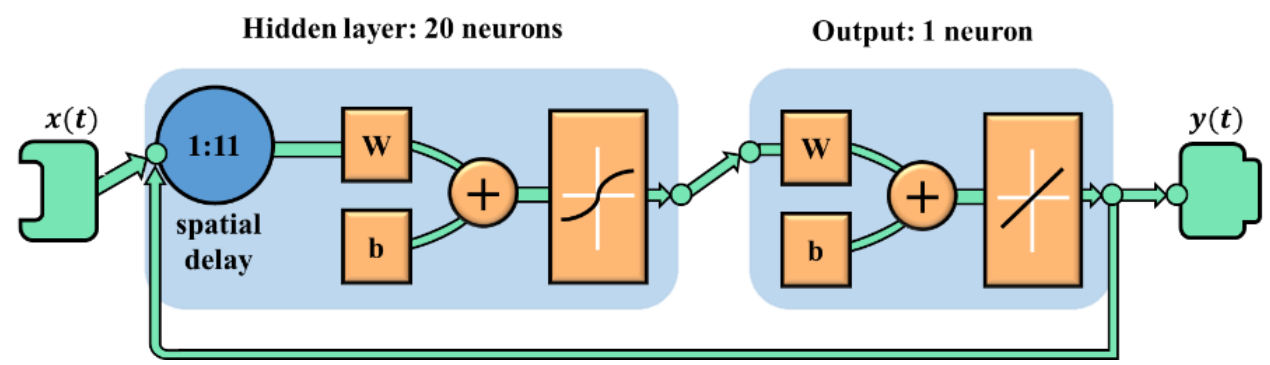

Figure 18. The prediction process of the NARNN.

In addition, two adaptive measures for the NARNN predictor were taken: 
For one thing, aiming at saltation tended to cause inaccurate prediction, especially those between level 4 and the others. One possible solution was to lessen the length of the prediction zone to $10 \mathrm{~m}$ when detecting such dramatic changes [29]. The demonstration of real-time feasibility is completed in Section 3.2.

For another, nerual networks trained by different training sets can be switched to better adapt to improve the generalization, when a certain pattern of the garbage distribution was recognized [30]. Different types of training sets could be obtained from the garbage model statistically established on the observations from different garbage condition roads.

Principal parameters of the NARNN predictor are listed in Table 4.

Table 4. Parameter setting of the NARNN.

\begin{tabular}{cc}
\hline Name & Value \\
\hline Hidden layer & 1 \\
Neurons in the hidden layer & 20 \\
Dependent past value/Time delay & 11 \\
Length of prediction zone & $20 \mathrm{~m} / 10 \mathrm{~m}$ (only regional) \\
\hline
\end{tabular}

To avoid the overlap, the NARNN took the remaining $25 \%$ of the data in the model as the test set. The prediction result of the NARNN is shown in Figure 19. Some magnified details are displayed in Figure 20 to highlight the predictor performance in particular regions.

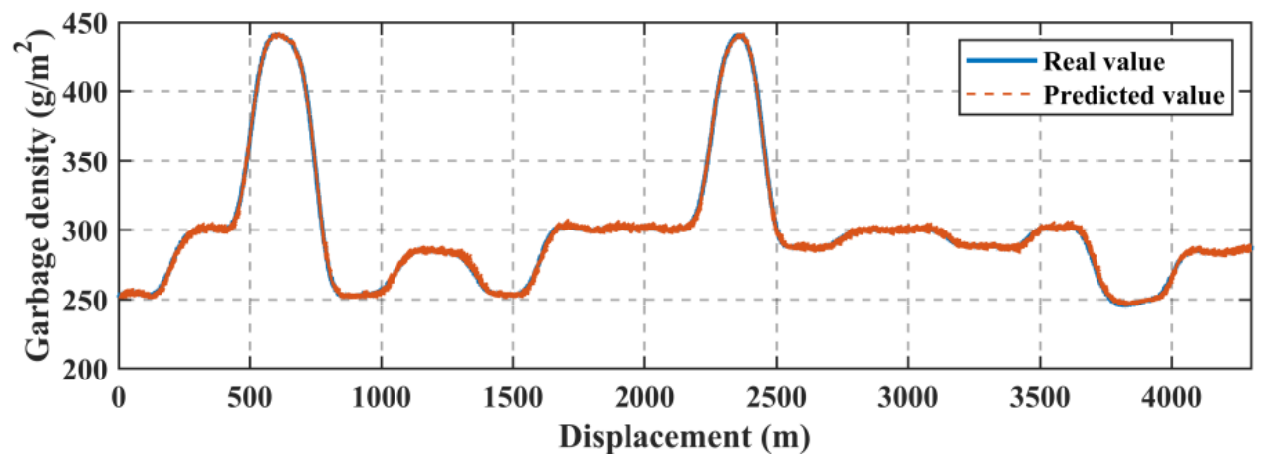

Figure 19. Prediction of the NARNN.
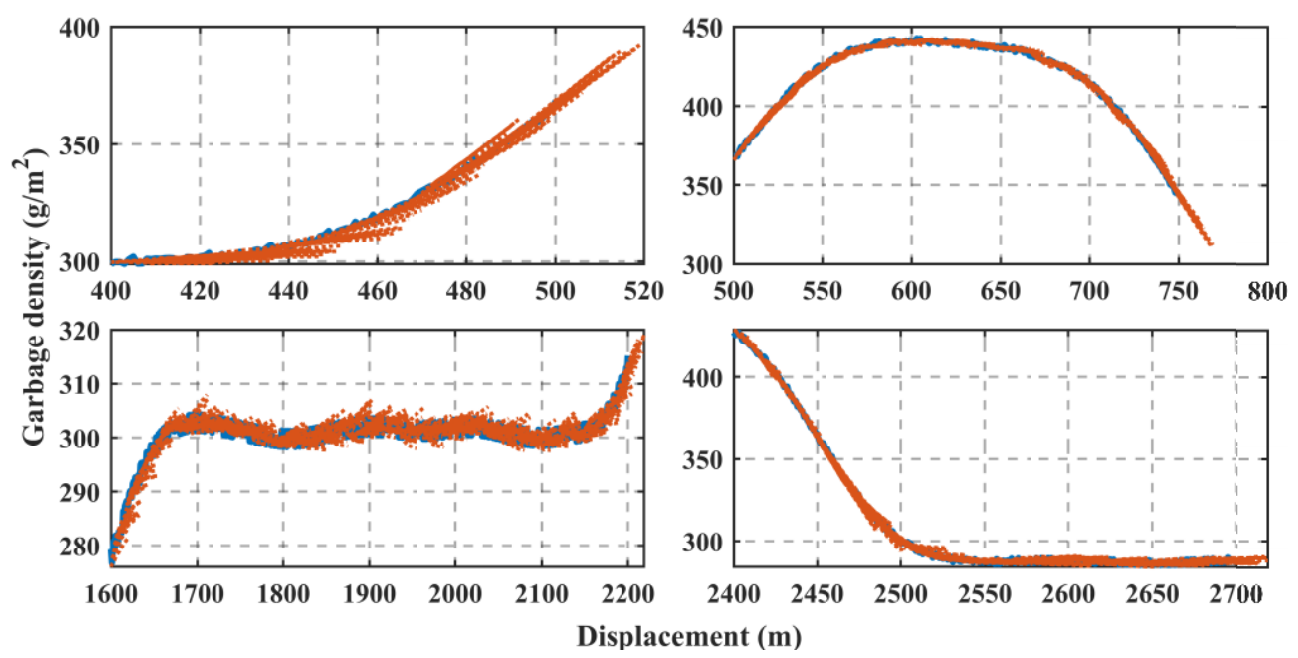

Figure 20. Magnified prediction details. 
Compared with the ARIMA model, which sometimes either overshot or dropped in advance [11], the NARNN seized the changing tendency of predicted series a little better. As Figure 20 shows, predicted values were a little more responsive and better anticipated the trends in the transitional areas from $400 \mathrm{~m}$ to $500 \mathrm{~m}$ or from $2400 \mathrm{~m}$ to $2600 \mathrm{~m}$, whereas in an area from $1600 \mathrm{~m}$ to $2200 \mathrm{~m}$, the range of predicted values better covered the real ones. In other words, the random fluctuations of real values in the flat distribution area were truly reflected.

For sanitation vehicles, such improvement might be critical because the constraints on garbage residue are so strict that a few wrong forecasts probably make the vehicle fail to accomplish clean tasks, which are manifested in Section 4.

Statistically, the mean and the maximum values of the root mean square error (RMSE, depicting deviations between real and predicted value) of all prediction zones were used to evaluate the performance of the NARNN, which is illustrated in Figure 21.

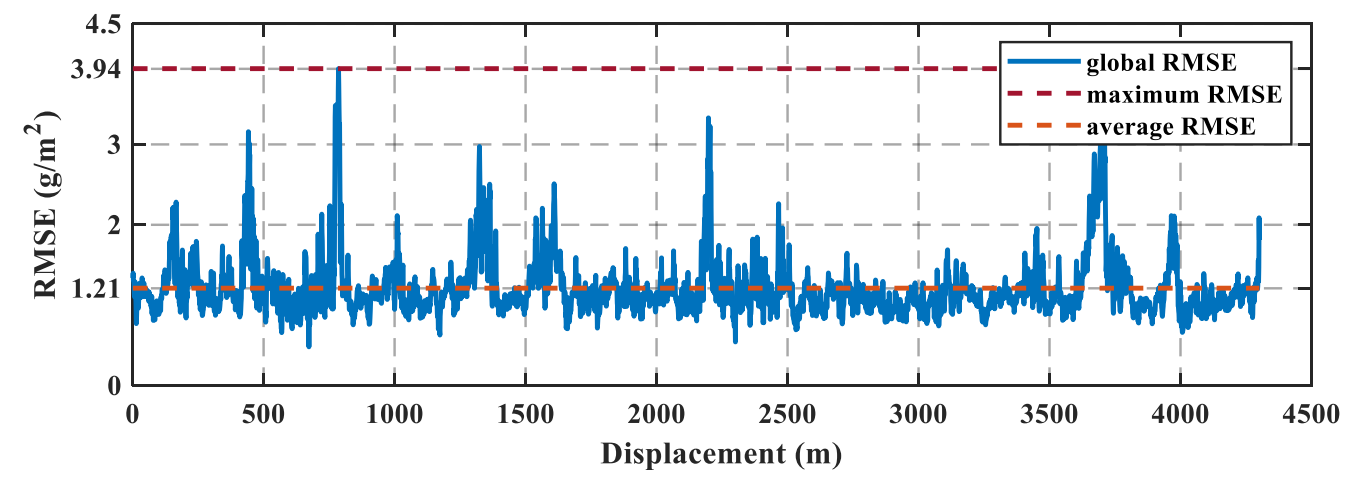

Figure 21. Prediction RMSE of the NARNN.

\subsection{The Framework of AMPC-Based EMS}

Endowed with the prediction ability by the NARNN predictor proposed above, an AMPC based EMS can be established to optimize the power distribution and ensure clean tasks. Therefore, a control-oriented system can be expressed as

$$
y=f(\vec{x}, \vec{u}, d)
$$

In the formulation, the vehicle acceleration $a_{v}\left(\mathrm{~m} / \mathrm{s}^{2}\right)$ and the rotating speed of the working motor $n_{w m}(\mathrm{rpm})$ are considered the control variables, noted as a vector $\vec{u}=\left[a_{v}, n_{w m}\right] ;$ the SoC of the battery, vehicle velocity $v(\mathrm{~km} / \mathrm{h})$, and working time $t(\mathrm{~s})$ are regarded as state variables, noted as a vector $\vec{x}=[S o C, v, t]$; and the garbage density $\delta\left(\mathrm{g} / \mathrm{m}^{2}\right)$ is considered a disturbance variable, noted as $d=\delta_{\text {predict }}$, which is predicted by the NARNN predictor.

With the spatial step $(\Delta x)$ set to $1 \mathrm{~m}$, for each spatial step, the discrete cost-to-go function $J_{n}$ is written as Equation (13):

$$
J_{n}=\sum_{n=k}^{k+L}\left(P_{w m}(n)+P_{d m}(n)\right) \cdot \frac{\Delta x}{1000 v(n)}
$$

where $J_{n}(\mathrm{~kW} \cdot \mathrm{h})$ represents the cost function on the prediction zone at the $n$ step, which minimizes the electricity consumption of the sanitation vehicle represented by that of the working motor and the driving motor; $L$ represents the length $(\mathrm{m})$ of the prediction zone; $v(n)$ represents the velocity $(\mathrm{km} / \mathrm{h})$ per spatial step; and the $P_{w m}(n)$ and the $P_{d m}(n)$ represent the electric power $(\mathrm{kW})$ consumed by the working motor and the driving motor per spatial step, respectively. As mentioned before, the former can be calculated by Equations (3) and (6), whereas the latter one can be obtained by Equation (2). 
Since the solution to this problem is a multistage process requiring a sequence of interrelated decisions, a local DP solver was implemented on the prediction zone to obtain optimal control series [30,31]. Based on the power requirement model (Equations (6) and (7)) and the predicted garbage, the DP optimized the cost function through relationships expressed as

$$
\begin{gathered}
v(k+1)=3.6 \cdot \sqrt{2 a(k) \Delta x+(v(k) / 3.6)^{2}} \\
\operatorname{SoC}(k+1)=-I_{b a t} \frac{3.6 \Delta x}{v(k) C_{b a t}}+\operatorname{SoC}(k)
\end{gathered}
$$

additionally, the parameters in the optimization process should be subject to the following constraints:

$$
\begin{gathered}
n_{d m \_l b} \leq n_{d m} \leq n_{d m \_u b} \\
n_{w m_{-} l b} \leq n_{w m} \leq n_{w m \_u b} \\
T_{d m \_l b} \leq T_{d m} \leq T_{d m \_u b} \\
T_{w m_{-} \_b} \leq T_{w m} \leq T_{w m \_u b} \\
I_{b a t \_l b} \leq I_{b a t} \leq I_{b a t \_u b} \\
V_{b a t}^{2}<4 R P_{d e \_b a t} \\
\psi_{\text {res }} \leq \psi_{\text {de_res }}
\end{gathered}
$$

where vectors $v(k), \operatorname{So} C(k)$, and $a(k)$ represent vehicle velocity, $\mathrm{km} / \mathrm{h}$, battery $S o C$, As, and vehicle acceleration, $\mathrm{m} / \mathrm{s}^{2}$ at spatial step $k$, respectively; $T_{d m \_l b}, T_{d m \_u b}, T_{w m \_l b}$, and $T_{w m \_u b}$ represent the lower and upper bound torque of the driving motor and the working motor, $\mathrm{N} \cdot \mathrm{m}$, respectively; $n_{d m_{-} l b}, n_{d m_{-} u b}, n_{w m_{\_} l b}$, and $n_{w w \_u b}$ represent the lower and upper bound rotating speed of the driving motor and the working motor, rpm, respectively; $I_{b a t}$ and $V_{b a t}$ represent the current and voltage of the battery; and $\psi_{\text {res }}$ and $\psi_{d e}$ res represent the residue and required residue of the garbage density, which was set to $13 \mathrm{~g} / \mathrm{m}^{2}$ referring to governmental regulations.

Setting the initial 11 spatial delays, the predictor continues the prediction process by updating the past 11 values with the detected real values. After having the first one of the acquired optimal control sequences performed, the vehicle updates state variables and repeats the whole process as moving to the next spatial step. The working principle of the AMPC-based EMS is illustrated in Figure 22.

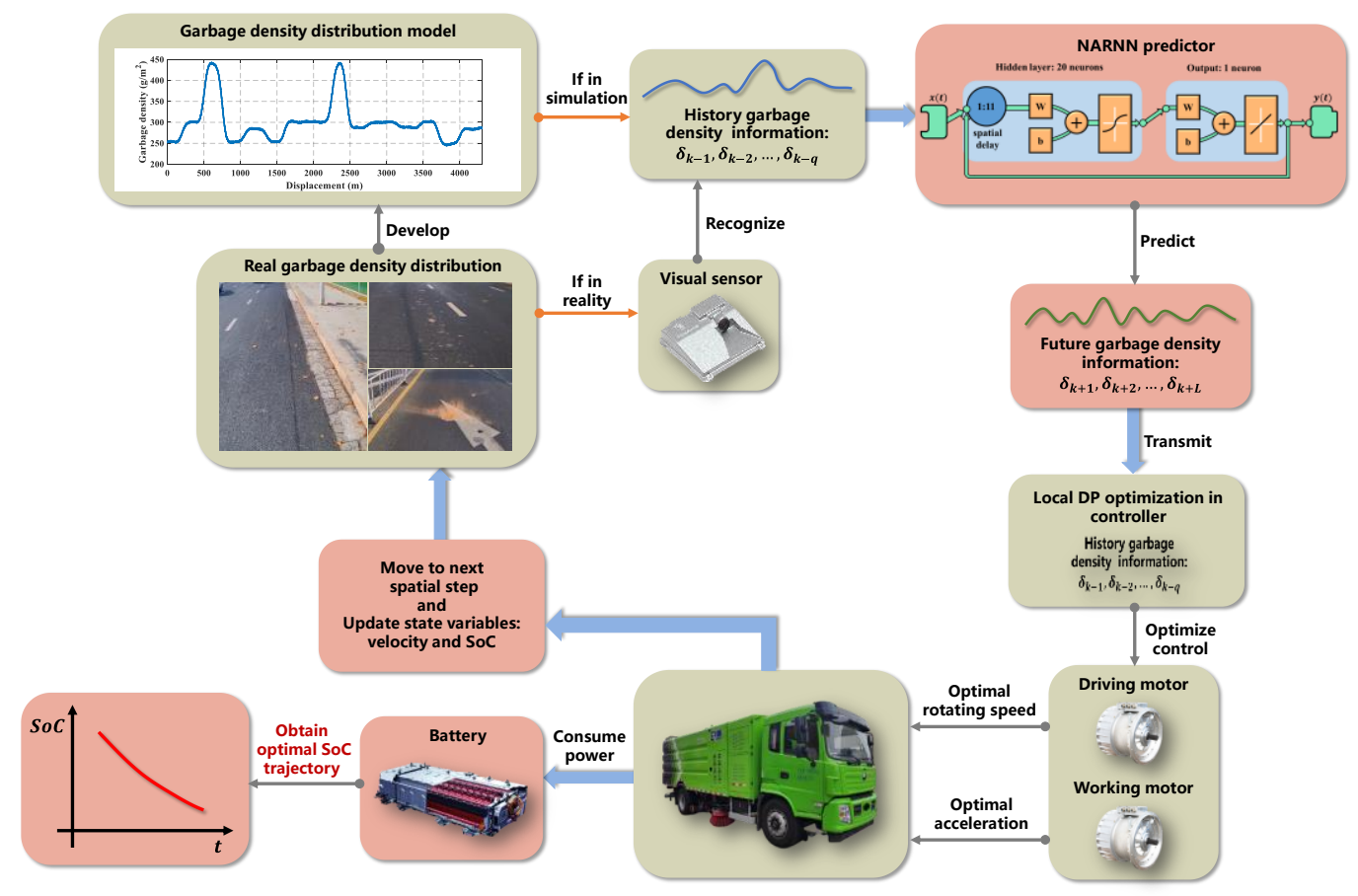

Figure 22. The AMPC based EMS. 
It is believed that the increase in the prediction zone length helps to improve global optimization but leads to a heavy computation burden. To testify the real-time implementation feasibility, the proposed AMPC-based EMS was implemented in an industrial personal computer (IPC, Inter Core i7-3610QM @2.3GHz) shown in Figure 23. The vehicle was assumed to travel at $8 \mathrm{~km} / \mathrm{h}$ due to almost constant velocity. Then, $20 \mathrm{~m}$ for the prediction zone equaled to $9 \mathrm{~s}$ on the time domain. The calculation time of the AMPC-based EMS on each prediction zone ranging from $0.3 \sim 0.45 \mathrm{~s}$ was far less than the predicted range ( $3 \%)$, which was also suitable for the shortened $10 \mathrm{~m}$ prediction zone because the calculating time was saved simultaneously. Thus, its real-time performance was well substantiated.

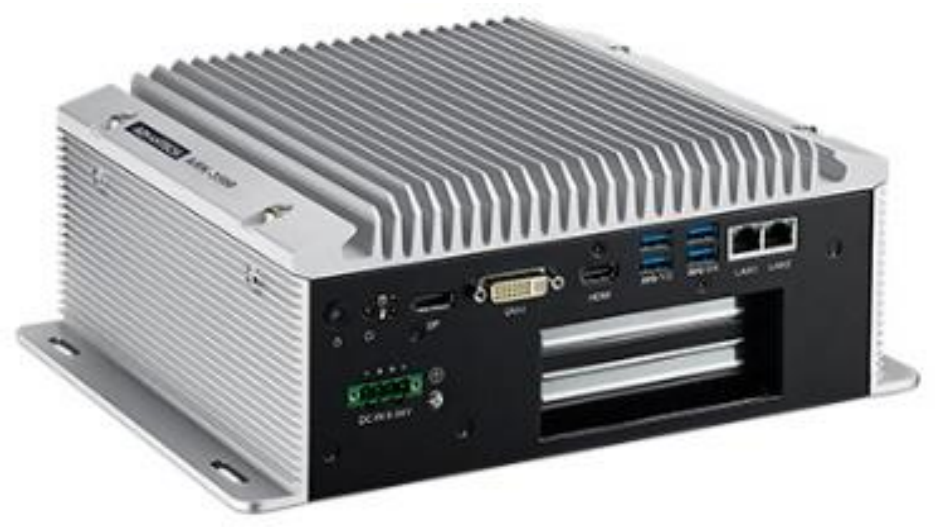

Figure 23. Industrial personal computer (ARK-3500).

Additionally, other existing EMSs, including the rule-based EMS and the DP-based EMS, were proposed to comprehensively evaluate the energy economy performance of the AMPC-based MPC.

\subsection{Other Existing EMSs}

First, a rule-based EMS was proposed based on the experience of drivers working on the target road. Those rules can be roughly summarized as two combinations of the vehicle velocity and the working motor rotating speed: One was $8 \mathrm{~km} / \mathrm{h}$ grouped with $1700 \mathrm{rpm}$; the other was $4 \mathrm{~km} / \mathrm{h}$ coupled with $1900 \mathrm{rpm}$. With visual judgment, drivers chose the former combination to deal with ordinary situations involving segments marked from levels 1 to 3 , whereas the latter one was especially executed to deal with situations that level 4 describes. Although well adapted to different scenarios, this conservative EMS was expected to realize more economic improvement.

The simple manipulation logic is illustrated in Figure 24.

Secondly, assuming the garbage density distribution is completely known, which uses the garbage model as a substitution, a DP-based EMS can be proposed. Applied to the global rather than the local prediction zone, the DP-based EMS had the same kinetic relationships and constraints as the local DP solver in the AMPC framework. Thus, the cost function of the DP-based EMS is expressed as

$$
J_{n}=\sum_{n=X_{0}}^{X_{n}}\left(P_{w m}(n)+P_{d m}(n)\right) \cdot \frac{\Delta x}{1000 v(n)}
$$

where $X_{0}$ and $X_{n}$ are the start and the final point of the solution domain decided by the garbage distribution model.

In the last section, the optimization result represented by the $\mathrm{SoC}$ variation of the DP-based EMS is used as a benchmark to evaluate the electricity consumption performance of the AMPC-based EMS. 


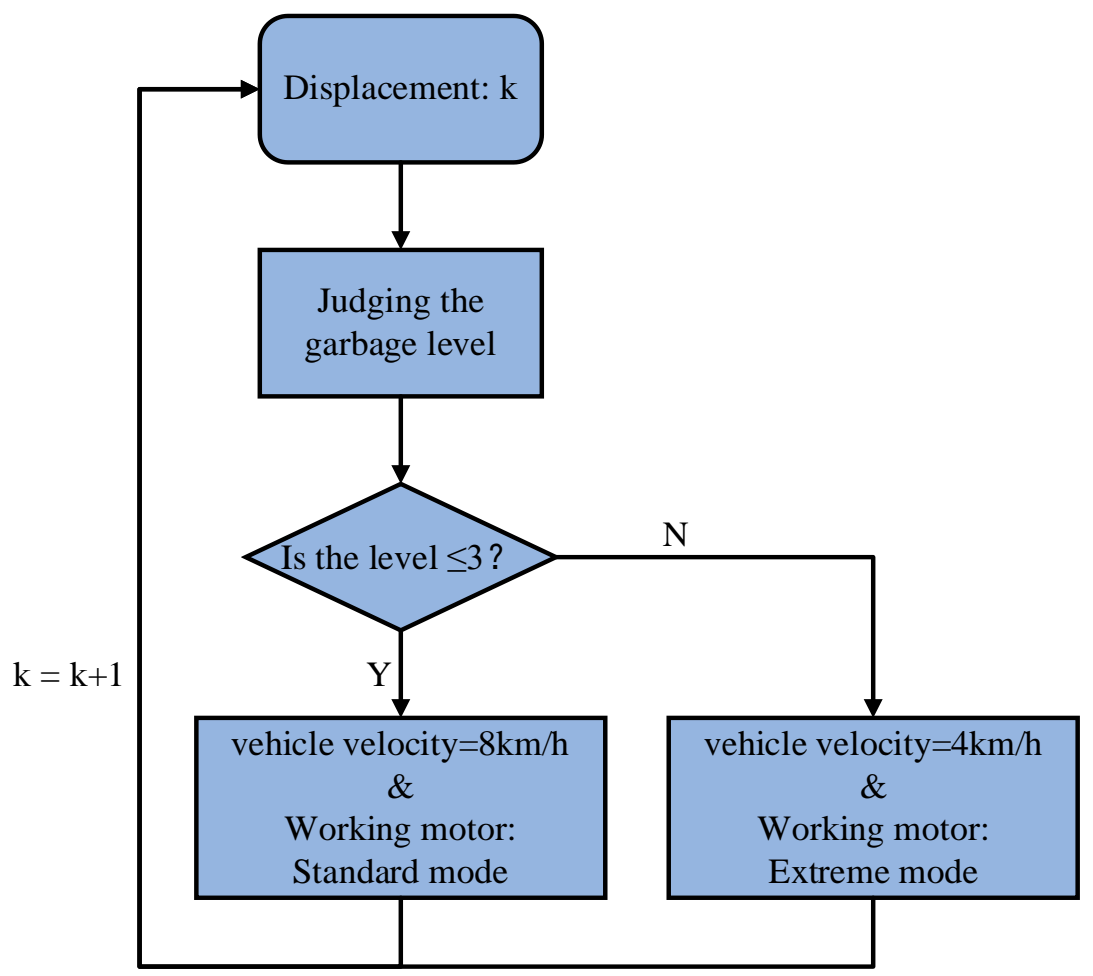

Figure 24. Logic of the rule-based EMS.

\section{Results and Discussions}

\subsection{Comparison of the Garbage Residue}

Figure 24 shows garbage residue distributions under different control strategies. Two fixed control variable combinations, which were the standard and the extreme mode of the working motor coupled with vehicle speed at $8 \mathrm{~km} / \mathrm{h}$, were also included in the comparison. Some critical details of Figure 24 are magnified in Figure 25.

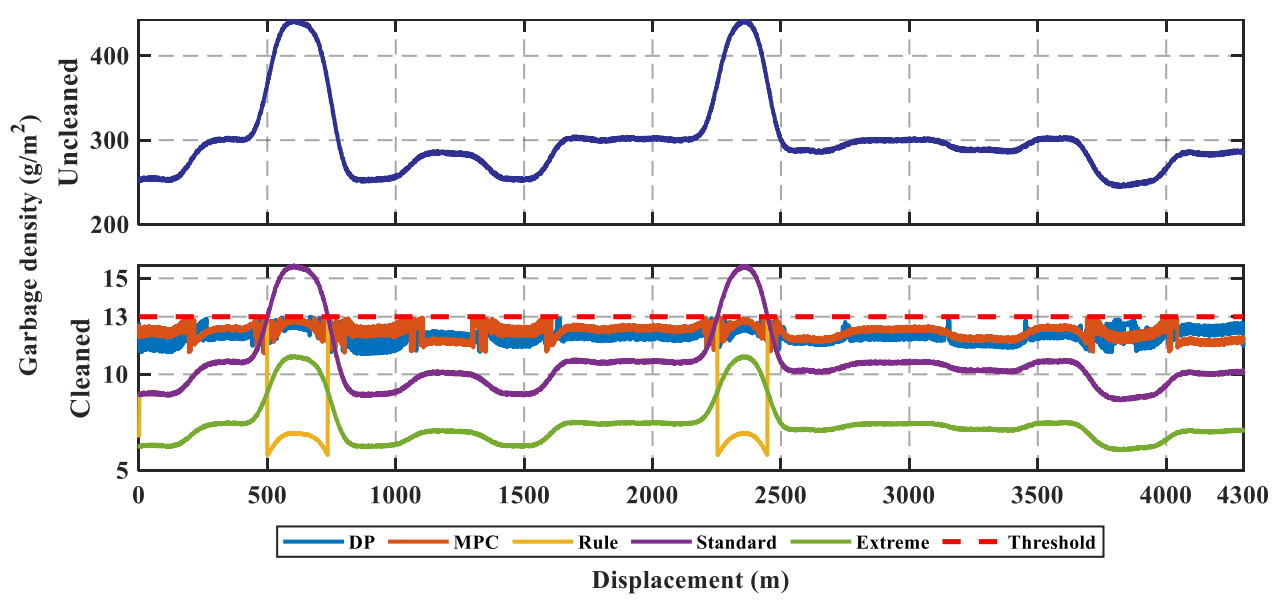

Figure 25. Garbage residues of different EMSs.

From the perspective of mission accomplishment, except for the fixed standard mode, all strategies met the requirement with varying degrees. It is noted that DP and the AMPC took full advantage of the regulation by restricting the residues within a narrow area very close to the threshold. By contrast, the rule-based strategy performed more desirably because of the low average of residues. Besides, there is no doubt that the fixed extreme mode can realize the best cleaning effect regardless of economic energy cost. 
Specifically, similar to the DP result, the adaptive AMPC strategy left residues a little higher and in a slenderer range, partly because the limited prediction zone disenabled the AMPC to foresee the garbage variation on a larger spatial scale. As mentioned in Section 3.1, in the parameter tuning process of the NARNN predictor, the AMPC occasionally had a few residues exceed the limit, manifesting the necessity of the accuracy improvement compared with the ARIMA model.

Statistical indicators for each strategy are summarized in Table 5, where the units are $\mathrm{g} / \mathrm{m}^{2}$.

Table 5. Main statistical indicators of different strategies.

\begin{tabular}{ccccc}
\hline Object Name & Standard Deviation & Mean & Maximum & Minimum \\
\hline Uncleaned garbage & 45.569 & 299.09 & 442.83 & 244.52 \\
Standard mode & 1.612 & 10.58 & 15.67 & 8.65 \\
Extreme mode & 1.127 & 7.40 & 10.96 & 6.05 \\
Rule-based strategy & 1.284 & 9.76 & 13 & 5.79 \\
DP based strategy & 0.383 & 12.01 & 12.99 & 11.05 \\
AMPC based strategy & 0.359 & 12.21 & 12.98 & 11.09 \\
\hline
\end{tabular}

\subsection{Comparison of the Electricity Consumption}

Figure 26 shows the SoC curves of different EMSs. It was manifested that the AMPC approximated DP perfectly. In terms of SoC final values, DP merely takes a slight advantage.
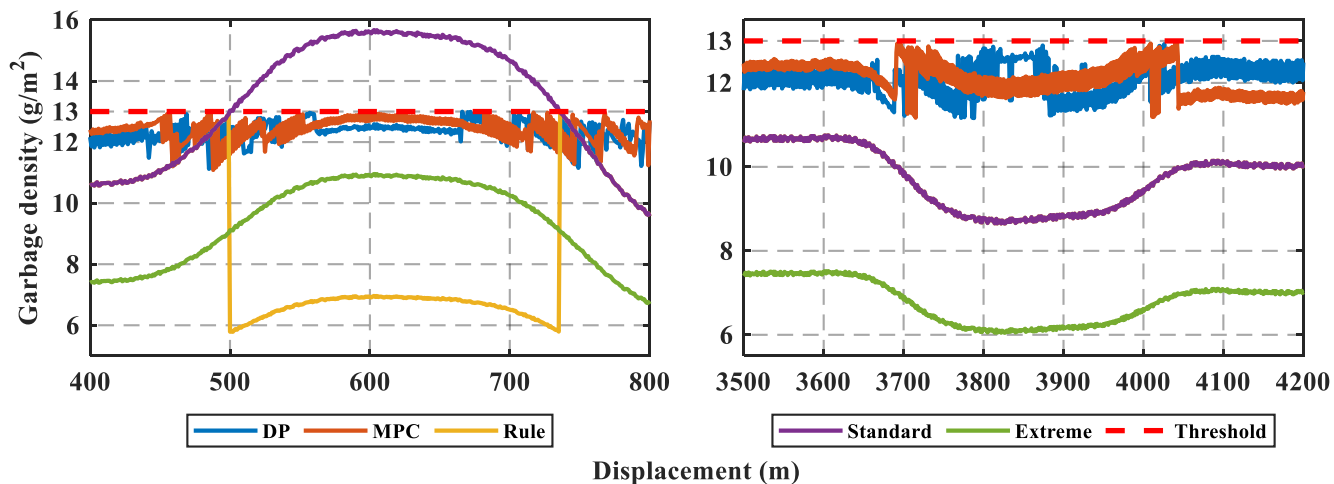

Figure 26. Magnified garbage residues.

Compared TO the rule-based strategy, optimization methods such as DP and the AMPC decreased battery SoC more evenly. Particularly, each time a sanitation vehicle encountered segments marked level 4, the expended electric energy increased dramatically, which is highlighted in the enlarged red rectangle. As a result, more segments that were difficult to clean up occurred on the road, and more energy was "wasted" by the rule-based strategy. For two fixed working modes, on the one hand, it is appropriate that the extreme mode consumed the most energy. On the other hand, it seems that the standard mode performed a little worse than DP but won on simplicity by making some sacrifices on garbage residue.

The results of the different strategies are evaluated in Table 6 with the rule-based one used as the baseline. 
Table 6. Energy consumption performance comparison of different strategies.

\begin{tabular}{ccc}
\hline Strategy & Consumed Energy $\mathbf{( k W h )}$ & Relative Improvement (\%) \\
\hline Rule-based & 32.44 & - \\
DP & 27.40 & +15.43 \\
Adaptive-AMPC & 27.43 & +15.53 \\
Fixed standard mode & 28.38 & +12.50 \\
Fixed extreme mode & 35.74 & -10.19 \\
\hline
\end{tabular}

\subsection{Comparison of Other Variables}

The distribution of optimal control variable sequences is shown in Figure 27. Compared with the MPC-based EMS, the rotating speed of working motor and velocity under DP control was more dispersed and had a slightly lower average, which reflects that DP tended to have the control variables change evenly and realize the global optimization because of grasping global information.

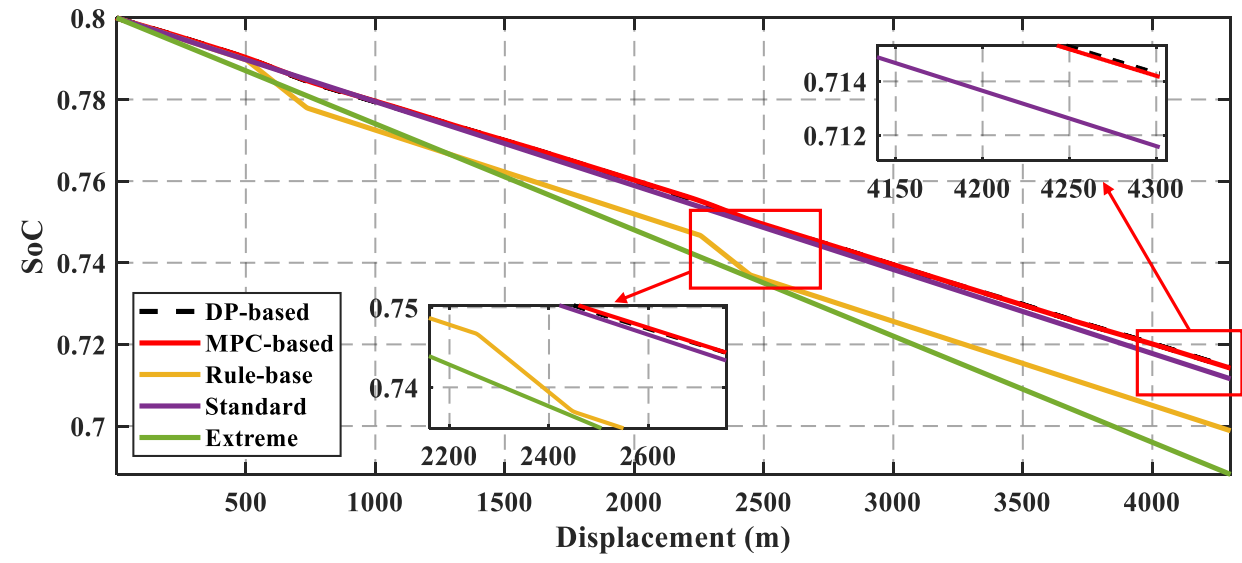

Figure 27. battery SoC variation of different EMSs.

As shown in Figure 28a, for one thing, the general mode of the working motor was dismissed by all three strategies, indicating that excessively low power of the working motor is not adequate for cleaning jobs in the target environment. For another, based on the distribution of optimization method, the regular rotating speed set at about $1700 \mathrm{rpm}$ in the rule-based strategy proved to be reasonable for a fixed deterministic strategy. However, optimal results indicated that more accurate and flexible adjustments ranging from $1675 \mathrm{rpm}$ to $1750 \mathrm{rpm}$ tend to be a better choice.

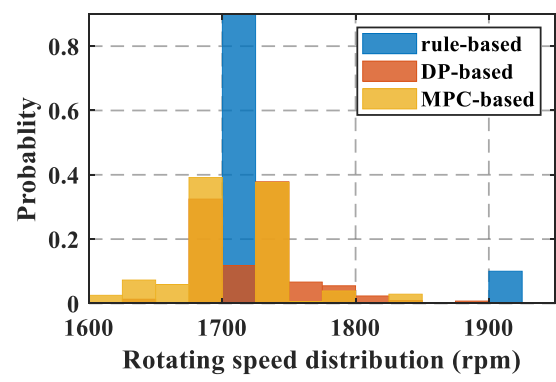

(a)

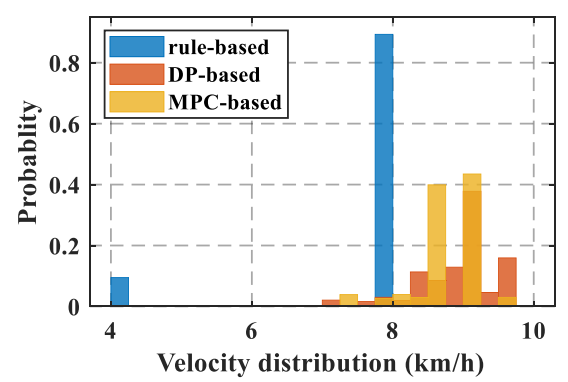

(b)

Figure 28. Distribution of the control variables: (a) rotating speed of the working motor and (b) velocity of the vehicle. 
In terms of velocity, which is shown in Figure 28b, both optimization strategies had a higher range than the rule-based one. Because higher velocity meant a greater discount on the cleaning effect of the working motor, there was redundant power of the rule-based strategy that could be spared. Hence, regular vehicle speed might be more desirably set at $8.5 \mathrm{~km} / \mathrm{h}$ for ordinary segments like level 1-level3, and at $7 \mathrm{~km} / \mathrm{h}$ for the extreme conditions. Moreover, due to a lack of flexibility on the adjustment of vehicle velocity, the rule-based strategy failed to decrease the consumed power in some regions as 500-700 m or 2200-2500 m, which are shown in Figure 29a, leading to unnecessary energy loss.

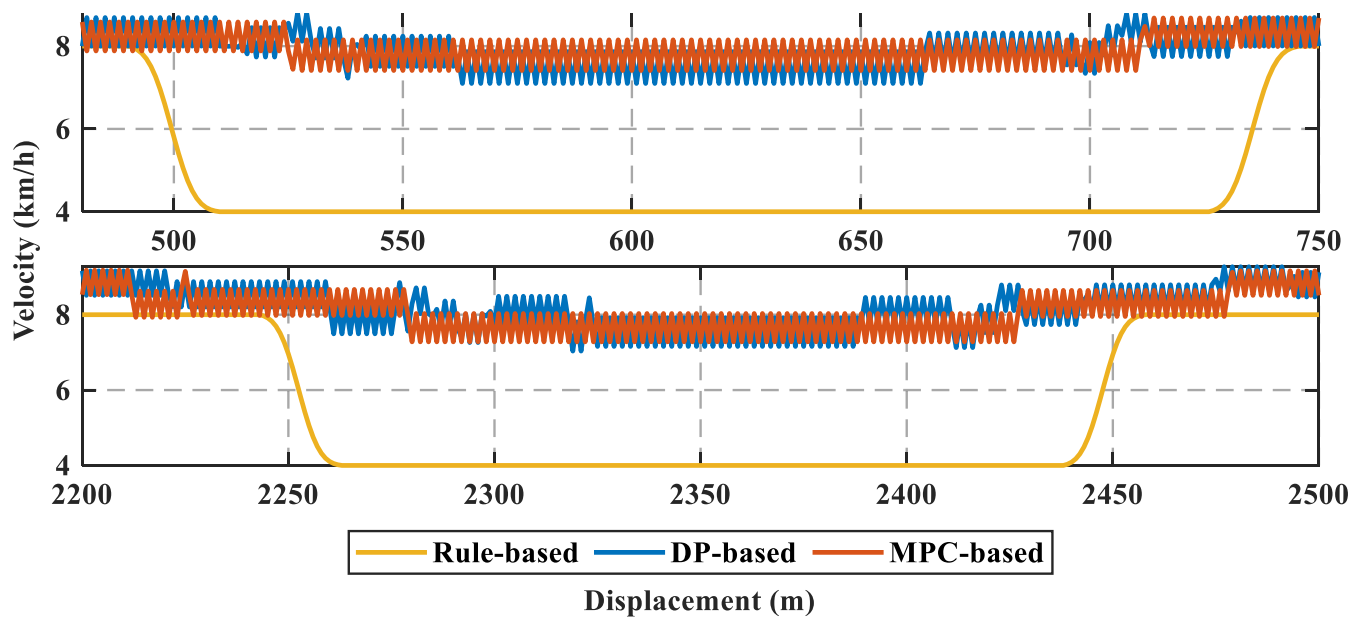

(a)

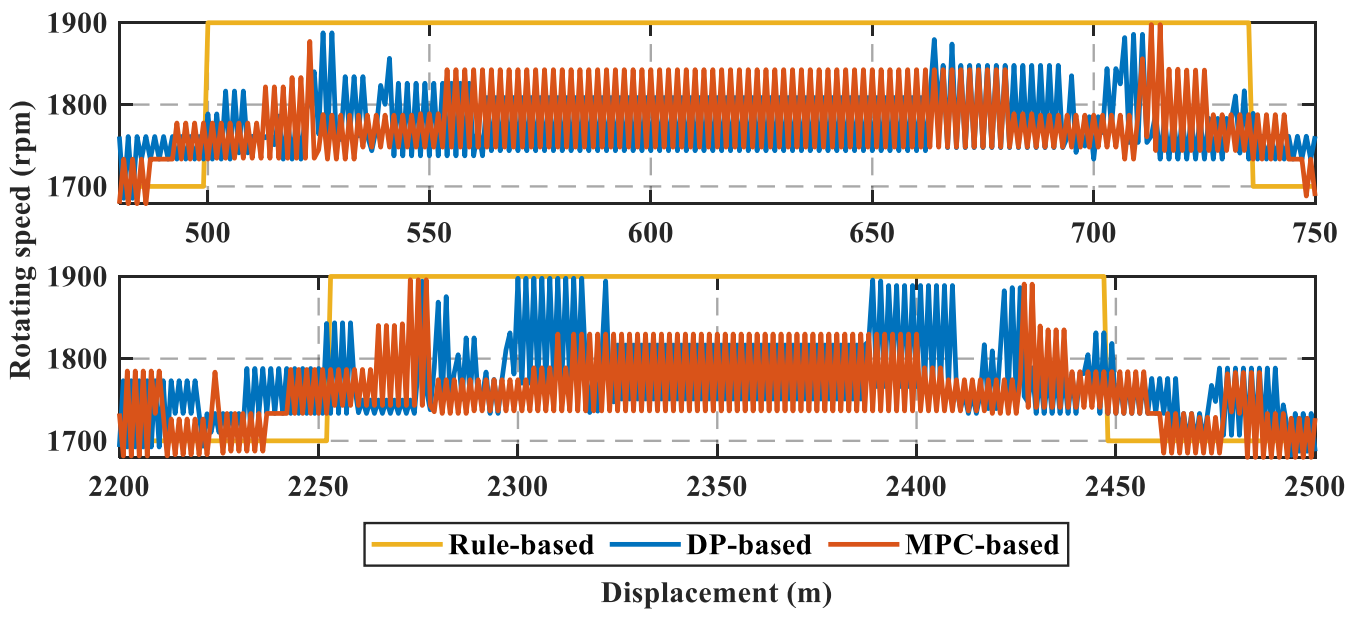

(b)

Figure 29. Magnified control variables: (a) vehicle velocity and (b) rotating speed of the working motor.

Details about vehicle operating states are shown in Figure 29. It is noted that the DPbased EMS performed a few more combinations of high rotating speed and low velocity than the AMPC, which explains the reason why the SoC under the AMPC decreased a little more slowly but had more garbage residue left than DP during the early stage in Figures 25 and 26.

Additionally, considering the sanitation vehicle equipped a full water-filled tank, frequent changes of acceleration should be avoided in pursuit of driving comfort. Hence, it is rational that both DP and the AMPC restricted acceleration to fluctuating slightly between $0.5 \mathrm{~m} / \mathrm{s}^{2}$ and $-0.5 \mathrm{~m} / \mathrm{s}^{2}$, as shown in Figure 30 . 

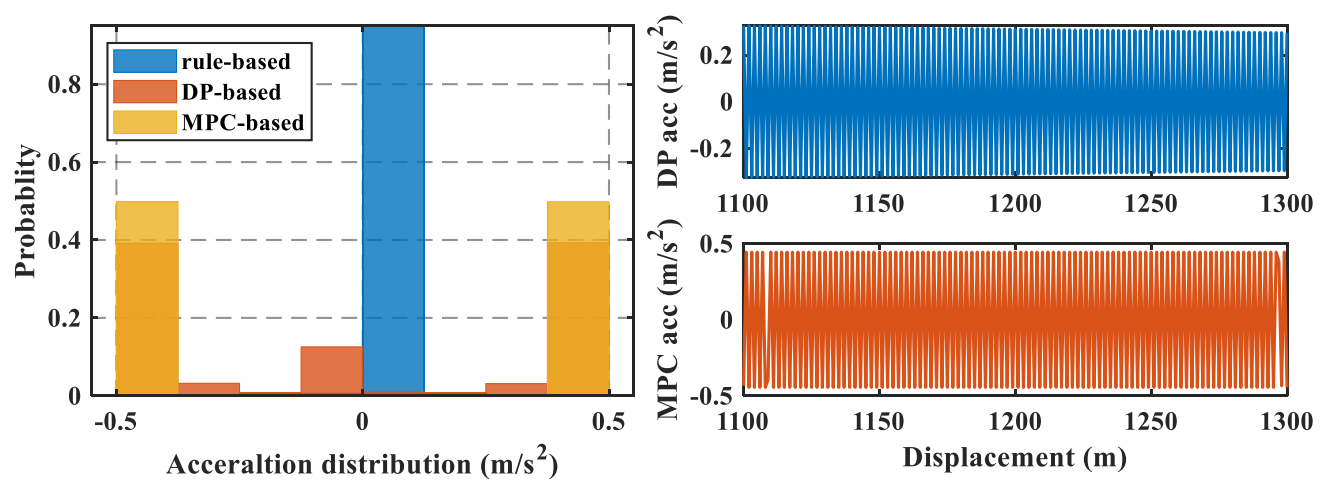

Figure 30. Acceleration of different EMSs.

As shown in Figure 28b, due to the higher average velocity, the AMPC proposed in this study lessened operating time from $35 \mathrm{~min}$ to $29 \mathrm{~min}$, which was a $16.7 \%$ improvement by contrast with the rule-based strategy. Time cost of each strategy is shown in Table 7.

Table 7. Time cost comparison.

\begin{tabular}{ccc}
\hline Strategy & Time Cost $(\min )$ & Time Improvement $(\%)$ \\
\hline Rule-based & 35.4 & - \\
DP-based & 29.0 & 18.1 \\
AMPC-based & 29.5 & 16.7 \\
\hline
\end{tabular}

Eventually, some guidelines for driver manipulations were concluded, such as reasonably adjusting regular driving speed to be a little higher and decreasing the use of the highest rotating speed of the working motor as much as possible. In addition, instead of pure manual operation, accurate and flexible manipulations of the real-time AMPC-based EMS could be realized with the assistance of automatic driving.

\section{Conclusions and Future Work}

The main conclusions can be drawn as follows:

1. Through the energy consumption analysis of principal devices onboard, it is concluded that the working motor and the driving motor were the main contributors, which took up to $89 \%$ of the total electricity consumption.

2. Based on the practical observation of the target route, a universal garbage distribution modeling method can be concluded, which covers almost all scenarios marked level 1 to level 4 .

3. A clean task-oriented AMPC-based EMS using the NARNN as the predictor is proposed, and its real-time feasibility was verified. Compared with the rule-based EMS, it was substantiated that the AMPC-based EMS saved electricity by $15.53 \%$, and well approximated the global optimization of the DP-based EMS. Additionally, the working time was lessened from $35 \mathrm{~min}$ to $29 \mathrm{~min}(17.14 \%)$ by the AMPC-based EMS.

Future work might focus on cleaning trajectory optimization for sanitation vehicles fleets or developing unmanned sanitation vehicles that automatically recognize the garbage type with visual sensors and better conduct manipulations obtained by optimized control strategies. Since the battery is aging with the practical application, the EMS considering battry state of health is also worthwhile to explore in future studies.

Author Contributions: Conceptualization, H.H. and H.W.; methodology, H.W.; software, H.W.; validation, H.W., J.L. and Y.B.; formal analysis, H.W.; investigation, H.W., B.Y.; resources, H.H., B.Y.; data curation, H.W.; writing-original draft preparation, H.W.; writing—review and editing, H.H., Y.C.; Y.B.; visualization, H.W.; supervision, H.H.; project administration, H.H.; funding acquisition, H.H.. All authors have read and agreed to the published version of the manuscript. 
Funding: This work was supported by the National key R\&D program of China (2018YFB0106200).

Institutional Review Board Statement: Not applicable.

Informed Consent Statement: Not applicable.

Data Availability Statement: Restrictions apply to the availability of these data. Data was obtained from Yutong Bus Co., Ltd and are available from Beizhan Yan with the permission of Yutong Bus Co., Ltd.

Conflicts of Interest: The authors declare no conflict of interest.

\section{References}

1. Yue, M.; Jemei, S.; Gouriveau, R.; Zerhouni, N. Review on health-conscious energy management strategies for fuel cell hybrid electric vehicles: Degradation models and strategies. Int. J. Hydrog. Energy 2019, 44, 6844-6861. [CrossRef]

2. Geetha, A.; Subramani, C. A comprehensive review on energy management strategies of hybrid energy storage system for electric vehicles. Int. J. Energy Res. 2017, 41, 1817-1834. [CrossRef]

3. Peng, J.; He, H.; Xiong, R. Rule based energy management strategy for a series-parallel plug-in hybrid electric bus optimized by dynamic programming. Appl. Energy 2017, 185, 1633-1643. [CrossRef]

4. Hofman, T.; Steinbuch, M.; van RM, D.; Serrarens, A. Rule-based energy management strategies for hybrid vehicles. Int. J. Electr. Hybrid Veh. 2007, 1, 71-94. [CrossRef]

5. Banvait, H.; Anwar, S.; Chen, Y. A rule-based energy management strategy for plug-in hybrid electric vehicle (PHEV). In Proceedings of the 2009 American Control Conference, St. Louis, MI, USA, 10-12 June 2009.

6. Wu, J.; Zou, Y.; Zhang, X.; Liu, T.; Kong, Z.; He, D. An online correction predictive EMS for a hybrid electric tracked vehicle based on dynamic programming and reinforcement learning. IEEE Access 2019, 7, 98252-98266. [CrossRef]

7. Zhang, S.; Xiong, R. Adaptive energy management of a plug-in hybrid electric vehicle based on driving pattern recognition and dynamic programming. Appl. Energy 2015, 155, 68-78. [CrossRef]

8. Yuan, Z.; Liu, T.; Sun, F.; Peng, H. Comparative study of dynamic programming and Pontryagin's minimum principle on energy management for a parallel hybrid electric vehicle. Energies 2013, 6, 2305-2318. [CrossRef]

9. Borhan, H.; Vahidi, A.; Phillips, A.M.; Kuang, M.; Kolmanovsky, I.V.; Cairano, S.D. MPC-based energy management of a power-split hybrid electric vehicle. IEEE Trans. Control Syst. Technol. 2011, 20, 593-603. [CrossRef]

10. Huang, Y.; Wang, H.; Khajepour, A.; He, H.; Ji, J. Model predictive control power management strategies for HEVs: A review. J. Power Sources 2017, 341, 91-106. [CrossRef]

11. Guo, J.; He, H.; Sun, C. ARIMA-based road gradient and vehicle velocity prediction for hybrid electric vehicle energy management. IEEE Trans. Veh. Technol. 2019, 68, 5309-5320. [CrossRef]

12. Zhang, Z.; He, H.; Guo, J.; Han, R. Velocity prediction and profile optimization based real-time energy management strategy for Plug-in hybrid electric buses. Appl. Energy 2020, 280, 116001. [CrossRef]

13. Sun, C.; Hu, X.; Moura, S.; Sun, F. Comparison of velocity forecasting strategies for predictive control in HEVs. In Proceedings of the Dynamic Systems and Control Conference, San Antonio, TX, USA, 22-24 October 2014; American Society of Mechanical Engineers: New York, NY, USA, 2014.

14. Sun, C.; Hu, X.; Moura, S.J.; Sun, F. Velocity predictors for predictive energy management in hybrid electric vehicles. IEEE Trans. Control Syst. Technol. 2014, 23, 1197-1204.

15. Xie, S.; He, H.; Peng, J. An energy management strategy based on stochastic model predictive control for plug-in hybrid electric buses. Appl. Energy 2017, 196, 279-288. [CrossRef]

16. Lin, C.-C.; Peng, H.; Grizzle, J. A stochastic control strategy for hybrid electric vehicles. In Proceedings of the IEEE,2004 American Control Conference, Boston, MA, USA, 30 June-2 July 2004.

17. Zhang, F.; Hu, X.; Langari, R.; Cao, D. Energy management strategies of connected HEVs and PHEVs: Recent progress and outlook. Prog. Energy Combust. Sci. 2019, 73, 235-256. [CrossRef]

18. Lin, Y.; Tang, P.; Zhang, W.J. Artificial neural network modelling of driver handling behaviour in a driver-vehicle-environment system. Int. J. Veh. Des. 2005, 37, 24-45. [CrossRef]

19. Vlahogianni, E.I.; Golias, J.C.; Karlaftis, M.G. Short-term traffic forecasting: Overview of objectives and methods. Transp. Rev. 2004, 24, 533-557. [CrossRef]

20. Park, J.; Chen, Z.; Kiliaris, L.; Kuang, M.L.; Masrur, M.A.; Phillips, A.M.; Murphey, Y.L. Intelligent vehicle power control based on machine learning of optimal control parameters and prediction of road type and traffic congestion. IEEE Trans. Veh. Technol. 2009, 58, 4741-4756. [CrossRef]

21. Bodin, L.; Fagin, G.; Welebn, R.; Greenber, J. The design of a computerized sanitation vehicle routing and scheduling system for the town of Oyster Bay, New York. Comput. Oper. Res. 1989, 16, 45-54. [CrossRef]

22. Zhao, D.; Wang, R. Research on parameters matching of electric sanitation vehicle powertrain. In Proceedings of the 2013 International Conference on Mechanical and Automation Engineering, Jiujang, China, 21-23 July 2013.

23. Gillespie, T.D. Fundamentals of Vehicle Dynamics; Society of Automotive Engineers: Warrendale, PA, USA, 1992 ; Volume 400. 
24. Bai, Y.; He, H.; Li, J.; Li, S.; Wang, Y.; Yang, Q. Battery anti-aging control for a plug-in hybrid electric vehicle with a hierarchical optimization energy management strategy. J. Clean. Prod. 2019, 237, 117841. [CrossRef]

25. Li, L.; You, S.; Yang, C.; Yan, B.; Son, J.; Chen, Z. Driving-behavior-aware stochastic model predictive control for plug-in hybrid electric buses. Appl. Energy 2016, 162, 868-879. [CrossRef]

26. Musardo, C.; Rizzoni, G.; Guezennec, Y.; Staccia, B. A-ECMS: An adaptive algorithm for hybrid electric vehicle energy management. Eur. J. Control 2005, 11, 509-524. [CrossRef]

27. Benrhmach, G.; Namir, K.; Namir, A.; Bouyaghroumni, J. Nonlinear Autoregressive Neural Network and Extended Kalman Filters for Prediction of Financial Time Series. J. Appl. Math. 2020, 2020, 5057801. [CrossRef]

28. Ruiz, L.G.B.; Cuéllar, M.P.; Calvo-Flores, M.D.; Jiménez, M.d.C.P. An application of non-linear autoregressive neural networks to predict energy consumption in public buildings. Energies 2016, 9, 684. [CrossRef]

29. Cao, J.; Peng, J.; He, H. Research on model prediction energy management strategy with variable horizon. Energy Procedia 2017, 105, 3565-3570. [CrossRef]

30. He, H.; Jia, H.; Sun, C.; Sun, F. Stochastic model predictive control of air conditioning system for electric vehicles: Sensitivity study, comparison, and improvement. IEEE Trans. Ind. Inform. 2018, 14, 4179-4189. [CrossRef]

31. Bellman, R. Dynamic programming. Science 1966, 153, 34-37. [CrossRef] [PubMed] 